\title{
The Role of Eddies in Buoyancy-Driven Eastern Boundary Currents
}

\author{
SuyAsh BiRE AND CHRISTOPHER L. P. WOLFE \\ School of Marine and Atmospheric Sciences, Stony Brook University, State University of New York, \\ Stony Brook, New York
}

(Manuscript received 3 March 2018, in final form 19 September 2018)

\begin{abstract}
An eastern boundary current (EBC) system driven by a large-scale meridional buoyancy gradient is simulated using an idealized eddy-resolving model. The EBC system consists of a pair of stacked meridional currents that flow poleward near the surface and equatorward at intermediate depths. Buoyancy advection in the EBC is primarily balanced by the shedding of eddies, with anticyclonic, warm-core eddies dominating near the surface and cyclonic, cold-core eddies found at intermediate depths. These boundary eddies play a significant role in both the eastern boundary circulation-by helping to trap the EBC near the coast-and the large-scale circulation through their effect on the downwelling limb of the overturning circulation. Momentum and thickness budgets analyzed using the thickness-weighted average framework highlight the role of eddy form drag in shaping and maintaining the EBC. The efficiency of the form drag increases dramatically at the offshore flank of the EBC. This zonal variation of the form drag is essential for maintaining a swift, narrow EBC. The essential physics of the EBC are illustrated using a simple, semianalytical model.
\end{abstract}

\section{Introduction}

Poleward surface currents along the eastern boundaries of the oceans are prominent features in the winter hemisphere. In the North Pacific and Atlantic they appear in the form of the Davidson Current (Hickey 1979) and the Iberian Poleward Current (Frouin et al. 1990; Haynes and Barton 1990). In the south Indian Ocean, the Leeuwin Current flows poleward throughout the year but is considerably stronger in the austral winter (Thompson 1984; Cresswell and Golding 1980). Modeling studies have shown that the Leeuwin Current and Iberian Poleward Current are forced by a large-scale meridional buoyancy gradient arising from differential heating over the subtropics (Godfrey and Weaver 1991; Peliz et al. 2003). The large-scale buoyancy gradient drives an eastward zonal flow at the surface, which causes water to pile up on the eastern boundary. The resulting zonal pressure gradient drives a geostrophic poleward current.

The large-scale meridional buoyancy gradient, which forces the poleward eastern boundary currents (EBCs), has little zonal structure, implying that the scale of these currents should be determined by internal dynamics. One would expect Rossby waves and eddies to radiate

\footnotetext{
Corresponding author: Suyash Bire, suyash.bire@stonybrook. edu
}

potential vorticity (PV) anomalies away from the eastern boundary, leaving behind very broad and slow currents. However, these currents are typically tens of kilometers wide and have speeds on the order of tens of centimeters per second. This fundamental questionwhat sets the narrow width of the buoyancy-forced currents-has yet to be conclusively answered.

The factors responsible for trapping these currents near the boundary are uncertain. Previous studies have attributed the trapping to bottom slope (Weaver and Middleton 1989, 1990; Csanady 1985; Furue et al. 2013; Benthuysen et al. 2014; Schloesser 2014) or high rates of entrainment and diapycnal mixing (McCreary 1981; McCreary et al. 1986; Pedlosky 2003; Pedlosky and Spall 2005). McCreary et al. (1992) suggested that Rossby waves emanating from the eastern boundary can be trapped by the background eastward flow. Cessi and Wolfe (2013, hereinafter CW13) found that, in eddy-resolving models, trapped EBCs arose in the adiabatic limit even with no bottom topography. They also found that Rossby wave propagation was not essential to their trapping mechanism.

All the eastern boundary currents are known to shed eddies. The observational studies of Griffiths and Pearce (1985), Pingree and Le Cann (1992), and Fang and Morrow (2003) found anticyclonic eddies being shed by surface poleward currents. Pelland et al. (2013) observed deep anticyclonic eddies shed by the California 
Undercurrent. Various modeling studies have been able to reproduce these rich eddy fields next to eastern boundaries. For example, Peliz et al. (2003) showed that the Iberian Poleward Current shed eddy pairs of opposite vorticity owing to the interaction of the current with the coastal topography. Kurian et al. (2011) investigated eddies shed by the California Current system, finding a greater prevalence of anticyclonic eddies at the depth of the poleward undercurrent. Molemaker et al. (2015) suggested that the interaction of bottom slope with the California Undercurrent was responsible for shedding anticyclonic eddies. Given this prevalence of eddies near the eastern boundaries of the oceans, it is not surprising that eddy processes played a dominant role in the trapping mechanism of CW13. In the present study, we observe a similar abundance of anticyclonic eddies near the eastern boundary; however, since we have straight coast and flat bottom, these eddies are formed by the instability of the EBC itself.

Following CW13, we use the thickness-weighted average (TWA) framework to study the effects of eddies on the EBC. The TWA framework was first proposed by De Szoeke and Bennett (1993) and refined by Young (2012) and Maddison and Marshall (2013). As the name suggests, the thickness-weighted average of a quantity is obtained by averaging over time along isopycnal layers after weighting the quantity with the layer thicknesses. The continuity and buoyancy equations combine to form a thickness equation, which is free of explicit eddy correlation terms. The TWA momentum equations contain the eddy correlations in the form of divergences of Eliassen-Palm (EP) flux tensors. Thus, the TWA system bundles the eddy correlation terms into the momentum equations and simplifies the eddy-mean flow interaction problem. The readers are referred to Young (2012) for mathematical notations and derivations.

CW13 found that the dominant momentum and PV balances in the EBC were between the Reynolds stress divergence and form drag. They argued that the former acted like a downgradient diffusion of thickness in the zonal direction, while the latter acted like a linear drag on meridional momentum. We will show that this balance is not, in itself, sufficient to produce a swift, narrow boundary current without accounting for the cross-shore distribution of form drag.

In the present study, we simulate a buoyancy forced $\mathrm{EBC}$ in an isopycnal-coordinate, eddy-resolving model with a flat bottom and low diapycnal diffusivity. The budgets of momentum, buoyancy, and thickness are analyzed in the TWA framework to determine the role played by eddies in boundary trapping. We expand on the idea presented in CW13 by showing that the zonal variation of the form drag is essential for producing a narrow EBC. The rest of this paper is arranged as follows: The model setup is described in section 2. The circulation observed in the model is described in section 3. Eddy shedding and its effect on the eastern boundary are discussed in sections 4 and 5 , respectively. Budgets of momentum and thickness are examined in section 6. Parameterizations for eddy momentum fluxes are explored in section 7, and the essential physics necessary for confining a narrow EBC are illustrated in section 8 using a simple semianalytical model. Finally, the results are summarized in section 9 .

\section{Model setup}

We use the Modular Ocean Model, version 6 (MOM6; a free and open-source model available at https://github.com/NOAA-GFDL/MOM6). MOM6 uses arbitrary Lagrangian/Eulerian coordinates, which allow it to seamlessly bridge the traditional divide between $z$-coordinate and isopycnal-coordinate ocean models (Adcroft and Hallberg 2006). Isopycnal coordinates are used for this study. The model is integrated forward in time using a mode-splitting algorithm (Hallberg and Adcroft 2009) with a baroclinic time step of $300 \mathrm{~s}$ and a barotropic time step determined at each forward integration. The continuity equation is solved using a piecewise-parabolic method and pressure-gradient force is accurately computed by combining an analytic formulation with the finite-volume method (Adcroft et al. 2008). Diapycnal diffusion is handled in accordance with Hallberg (2000).

The horizontal model domain is a Northern Hemisphere sector running from $-25^{\circ}$ to $0^{\circ}$ in the zonal direction and from $10^{\circ}$ to $60^{\circ} \mathrm{N}$ in the meridional direction. The depth of the model $H$ is uniform at $3000 \mathrm{~m}$. The horizontal grid spacing is uniformly $1 / 25^{\circ}$, which gives a uniform meridional grid resolution of $4.3 \mathrm{~km}$ and a zonal grid resolution decreasing from $4.3 \mathrm{~km}$ at the southern boundary to $2.2 \mathrm{~km}$ at the northern boundary. The high horizontal resolution ensures that the model resolves mesoscale eddies and their momentum and buoyancy fluxes. We use 30 isopycnal layers, uniformly spaced in buoyancy.

Salinity in the model is kept constant, and we use the linear equation of state so that buoyancy $b$ is related to temperature $\theta$ by

$$
b=\frac{d b}{d \theta}\left(\theta-\theta_{0}\right),
$$

where

$$
\frac{d b}{d \theta}=2 \times 10^{-3} \mathrm{~m} \mathrm{~s}^{-2} \mathrm{~K}^{-1}
$$




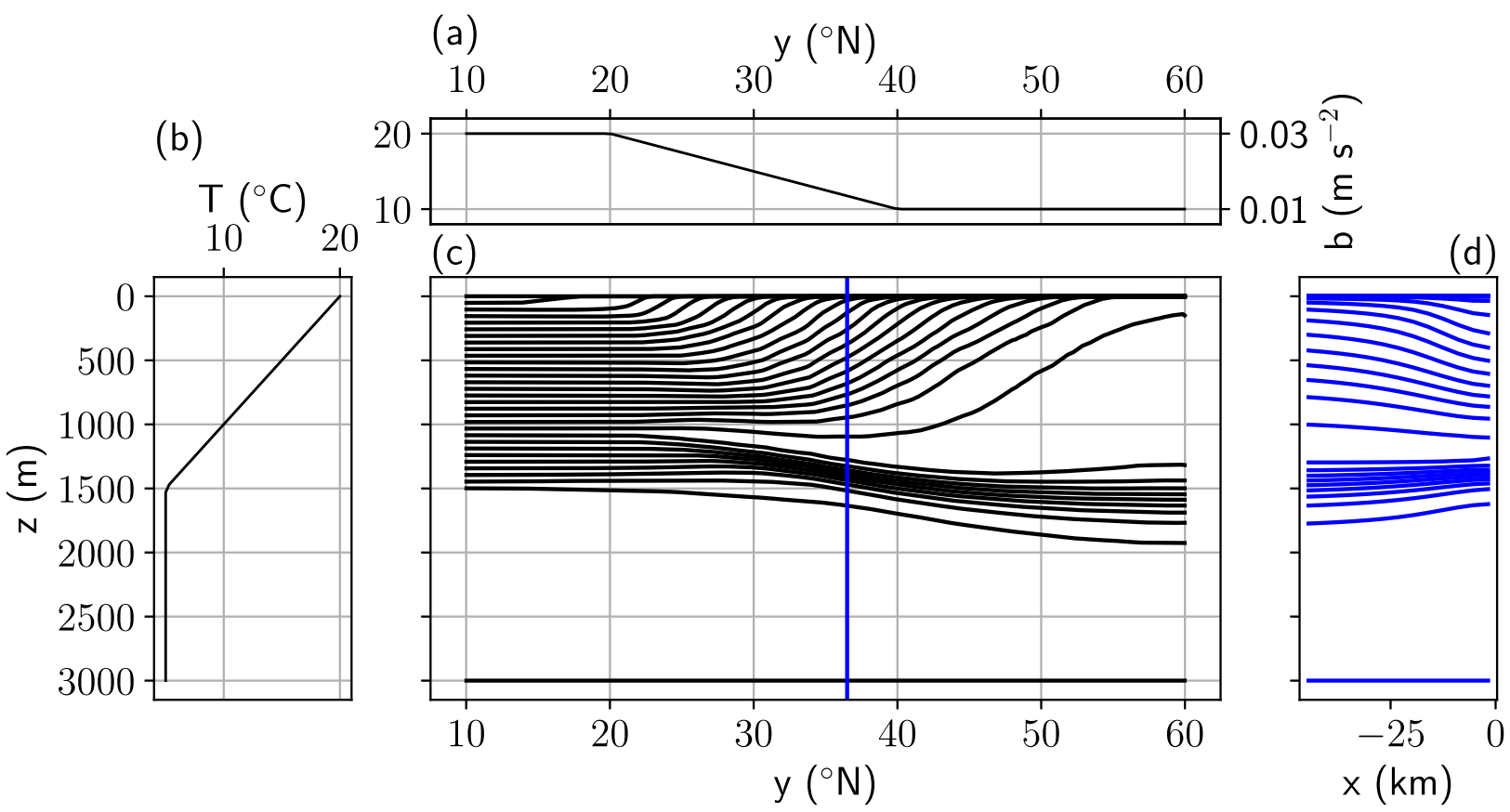

FIG. 1. Forcing and mean state of the model. (a) Relaxation target for surface buoyancy. (b) Relaxation target for southern boundary buoyancy. (c) Meridional section showing the mean height of the isopycnal layers averaged over $0.5^{\circ}$ closest to the eastern boundary. (d) Zonal section showing the mean height of the isopycnal layers near the eastern boundary at $36^{\circ} \mathrm{N}$, indicated by the blue line in (c).

and $\theta_{0}=5^{\circ} \mathrm{C}$. At the southern edge of the domain, the temperature in the upper $1500 \mathrm{~m}$ is relaxed to the background state with a constant buoyancy frequency:

$$
N^{2} \stackrel{\text { def }}{=} b_{z}=2 \times 10^{-5} \mathrm{~s}^{-2},
$$

where the subscript denotes partial differentiation. Below $1500 \mathrm{~m}$, the temperature is relaxed to $\theta_{0}$ (Fig. $1 \mathrm{~b}$ ). The relaxation occurs in a sponge layer that extends $4^{\circ}$ from the southern boundary. The relaxation time scale is 1 day $^{-1}$ in the southernmost $2^{\circ}$ and then decreases linearly to zero over the next $2^{\circ}$. flux

The model is forced at the surface by the buoyancy

$$
F=w^{*}\left(b^{*}-b_{\text {surf }}\right),
$$

where $w^{*}=4 \mathrm{~m}$ day $^{-1}, b_{\text {surf }}$ is the buoyancy averaged over the uppermost $1 \mathrm{~m}$, and $b^{*}$ is the zonally uniform relaxation target, given by

$$
b^{*}= \begin{cases}b_{\max }, & y \leq 20^{\circ} \mathrm{N}, \\ b_{\max }-\frac{\Delta b}{20^{\circ} \mathrm{N}}\left(y-20^{\circ} \mathrm{N}\right), & 20^{\circ} \mathrm{N}<y \leq 40^{\circ} \mathrm{N}, \\ b_{\max }-\Delta b, & y>40^{\circ} \mathrm{N},\end{cases}
$$

where $b_{\max }=0.03 \mathrm{~m} \mathrm{~s}^{-2}$ and $\Delta b=0.02 \mathrm{~m} \mathrm{~s}^{-2}$ (Fig. 1a). This profile is similar to that used by McCreary et al. (1986).

Dissipation is provided by both a Laplacian horizontal viscosity (coefficient $A_{H}=10 \mathrm{~m}^{2} \mathrm{~s}^{-1}$ ) and Smagorinsky biharmonic viscosity with a coefficient of 0.06 , which is used to remove vorticity at the grid scale without damping the large-scale flow (Griffies and Hallberg 2000). The vertical viscosity and diapycnal diffusivity are both set to $10^{-4} \mathrm{~m}^{2} \mathrm{~s}^{-1}$. The lateral boundary conditions are no slip. Quadratic drag is specified in a 10-m-thick bottom boundary layer with a drag coefficient of $3 \times 10^{-3}$. A crude representation of the mixed layer is provided by elevating the vertical viscosity in the top $20 \mathrm{~m}$ to $10^{-2} \mathrm{~m}^{2} \mathrm{~s}^{-1}$; however, this layer has little effect on the present results since there is no momentum forcing at the surface. The model is integrated until a statistically steady state is achieved ( $~ 35$ years). It should be noted that eddy-resolving models tend to equilibrate faster than coarse-resolution models because eddy stirring leads to faster transfer of surface information to the deep ocean along outcropping isopycnals. Unless otherwise stated, the figures in the following sections are averaged over a 10-yr period from year 38 to 47 .

\section{Description of the circulation}

Figure 2 shows the meridional overturning circulation (MOC) for the residual flow, integrated along isopycnals. 


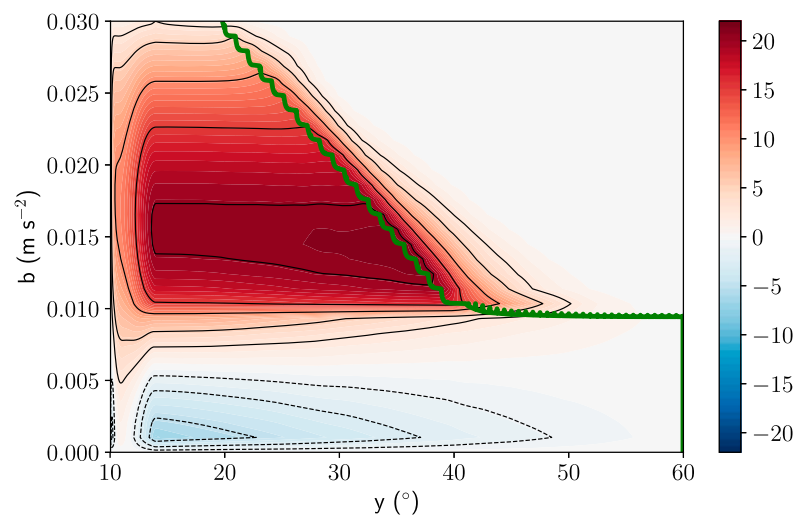

FIG. 2. Meridional overturning streamfunction for the residual flow ( $\mathrm{Sv} ; 1 \mathrm{~Sv} \equiv 10^{6} \mathrm{~m}^{3} \mathrm{~s}^{-1}$ ) as a function of latitude and buoyancy (contours and shading; contours are drawn at -5 , $-2.5,-1,2.5,5,10,15$, and $20 \mathrm{~Sv})$. The sense of the circulation is clockwise around highs. Isopycnals above the green line lie in the swash zone.

Isopycnals above the green line are in contact with the surface at least $1 \%$ of the time; following CW13, we refer to this region as the swash zone. Isopycnals in the swash zone are intermittently exposed to diabatic forcing at the surface. For the residual MOC, streamlines crossing isopycnals indicate diapycnal flow. Nearly all diapycnal flow in the upper cell is confined to either the southern relaxation zone, where dense water is made lighter, or the swash zone, where light water is made denser. Thus, the flow in this cell away from the surface is very nearly adiabatic as diapycnal diffusion plays a negligible role. In contrast, the diapycnal flow outside the southern relaxation zone in the weak counterclockwise bottom water cell is driven entirely by diapycnal diffusion.

We define streamfunctions for the zonal overturning circulation (ZOC) due to the mean and residual circulation as

$$
\Psi^{\sharp}(\tilde{x}, \tilde{b})=-\int_{0}^{\tilde{b}} \int \bar{\sigma} \bar{u} d \tilde{y} d b^{\prime}
$$

and

$$
\Psi(\tilde{x}, \tilde{b})=-\int_{0}^{\tilde{b}} \int \overline{\sigma u} d \tilde{y} d b^{\prime}
$$

respectively, where the tildes represent isopycnal coordinates, the bar indicates averaging in time along isopycnals, and $\sigma$ represents the thickness of isopycnal layers. A more formal introduction to the mathematical notation is given in section 5 . The meridional integration is over the full latitudinal range of the domain. As with the MOC, contours of $\Psi$ that cross isopycnals indicate diapycnal flow. In contrast, contours of $\Psi^{\sharp}$ that cross isopycnals indicate mean flow normal to mean isopycnals; in the limit of small slopes, this is approximately equivalent to the mean vertical velocity.

The mean and residual ZOCs are shown in Figs. 3a and $3 \mathrm{~b}$, respectively. While both ZOCs give a similar overall sense of the circulation-a strong clockwise overturning circulation within the swash zone over a weak counterclockwise bottom cell-they differ dramatically near the eastern boundary. The mean ZOC has strong upwelling within a western boundary layer balanced primarily by strong downwelling in an eastern boundary layer, with weak downwelling throughout the interior. In particular, the upwelling along the western boundary is stronger than the downwelling on the eastern boundary, in agreement with the results of Pedlosky and Spall (2005). The eastern downwelling boundary layer is entirely absent in the residual ZOC, and transformation of dense water to lighter water along the western boundary is balanced entirely by a broadly distributed densifying transformation throughout the interior of the domain. Thus, while the western boundary is a site of vigorous upwelling and water mass transformation, the eastern boundary does not contribute significantly to water mass transformation. The difference between the two pictures is due to eddies, which induce an eastern boundary circulation that cancels the mean downwelling almost perfectly.

Taken together, the ZOCs and the residual MOC (which does not differ qualitatively from the mean MOC) suggest a 3D residual circulation where water upwells diabatically in the southwestern corner of the domain and spreads nearly adiabatically along shoaling isopycnals. Once the water reaches the swash zone, it is converted into denser water that returns-again, nearly adiabatically-to the southern boundary. The difference between the two ZOCs indicates that the actual trajectories of water parcels are more complicated than the residual flow suggests, with significant vertical excursions along the eastern boundary that do not result in water mass transformation. This decoupling between the locations of downwelling and transformation has been noted previously by Spall and Pickart (2001).

The mean isopycnals close to the eastern boundary are shown in Fig. 1c. The background stratification is set in the southern part of the domain where the isopycnals are nearly flat. Isopycnals begin to shoal northward of $20^{\circ} \mathrm{N}$ to accommodate the surface forcing. The relaxation at the southern boundary mimics the stratification set at the Southern Ocean (Wolfe and Cessi 2010; Nikurashin and Vallis 2011), while relaxation at the surface represents the interaction with a zonally uniform atmosphere (Haney 1971).

The SST mostly follows the meridional pattern of the imposed surface relaxation, except that the eastern half 
(a)

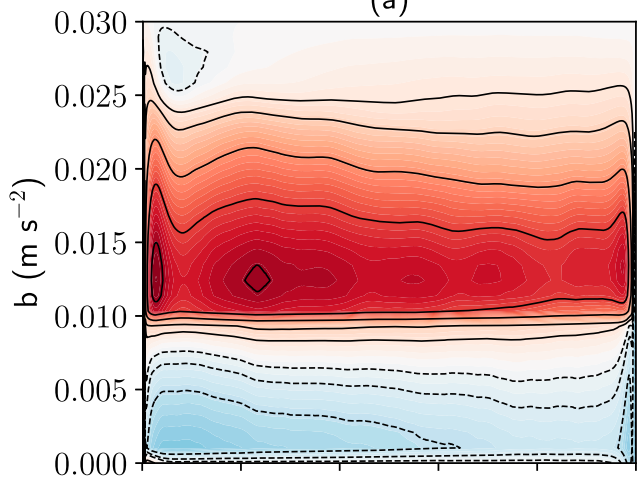

(b)

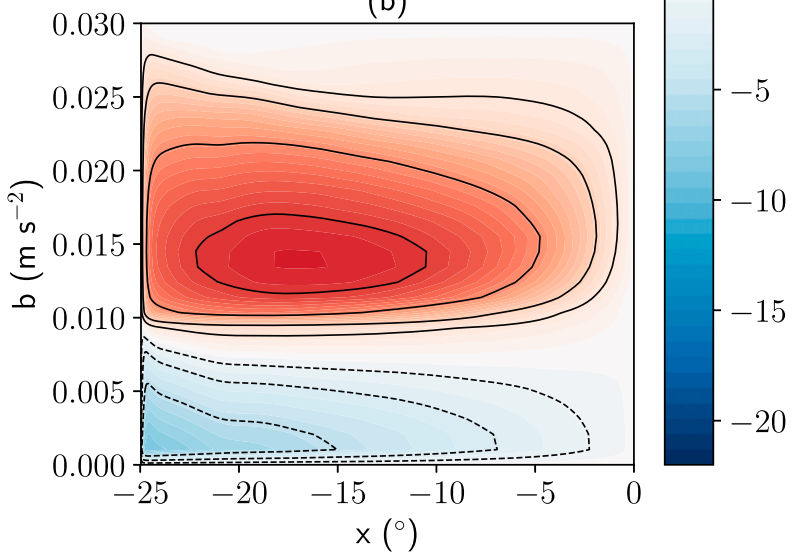

FIG. 3. Zonal overturning streamfunction for the (a) mean and (b) residual flow (Sv) as a function of longitude and buoyancy (contours and shading; contours are drawn at $-5,-2.5,-1,2.5,5$, $10,15$, and $20 \mathrm{~Sv})$. The sense of the circulation is clockwise around highs.

of the domain is warmer than the surface relaxation target (Fig. 4a) owing to two factors: 1) meridional advection of warm water by the poleward $\mathrm{EBC}$ and 2) warm-core eddies shed from the eastern boundary (more on this in section 5). The surface flow follows the SSH contours (Fig. 4a), flowing northward in a baroclinic western boundary current that diverges from the coast near $28^{\circ}$ and drifts east-by-northeastward across the basin. This flow downwells when it impinges on the eastern boundary and the resulting isopycnal deflection drives a poleward surface jet between $25^{\circ}$ and $55^{\circ} \mathrm{N}$. The flow at intermediate depths $(\sim 1000 \mathrm{~m})$ is similar to the surface flow but with the circulation reversed. A southward eastern boundary undercurrent is supported by the compressed isopycnals found near $1000 \mathrm{~m}$ along the eastern boundary (Fig. 1d). Water from the undercurrent detrains from the $\mathrm{EBC}$ and forms the intermediate-depth westward flow, which feeds into a southward deep western boundary current. In the Eulerian sense, the circulation is closed by connecting the surface and $1000 \mathrm{~m}$ circulation by upwelling and downwelling at the western and eastern boundaries, respectively (cf. Fig. 3a).

The snapshots of SST and vertically averaged vorticity (Figs. 4a,b), as well as the vertically averaged eddy kinetic energy (EKE; Fig. 4c) attest to the prevalence of eddies in the flow: these highly energetic mesoscale features occupy the entire region of eastward surface flow as well as a wedge near the eastern boundary north of $40^{\circ}$. As will be discussed in section 4 , many of the eddies form on the eastern boundary. A considerable fraction also arise from the western boundary current or are formed spontaneously in the band of westward surface flow, which is supercritical with respect to baroclinic instability.

Wind forcing is absent in this model and the thick bottom layer (cf. Fig. 1) insulates the upper-ocean flow from the bottom drag; the barotropic flow (not shown) is therefore weak and disorganized compared to the baroclinic flow. The exceptions are two inertial recirculation gyres near the separation point of the western boundary current. These gyres only extend $5^{\circ}$ from the western boundary and do not significantly affect the interior and eastern boundary flow.

The slope of the outcropping isopycnals in Fig. 1c is smaller than that in previous studies using $z$-coordinate models (e.g., de Verdière 1988; Marotzke 1997; Sumata and Kubokawa 2001; Cessi and Wolfe 2013). This difference can be attributed to the difference in the way convection is handled in $z$-coordinate models versus isopycnal-coordinate models (Park and Bryan 2001). Height-coordinate models produce excessive downwelling near the eastern boundary at the expense of northward transport. In contrast, the isopycnalcoordinate model in the present study produces a current that flows northward along the upward-sloping isopycnals. In the Eulerian-mean vertical velocity, this appears as an upwelling in the upper $500 \mathrm{~m}$ of the water column (see section 5). Despite the difference in the near-surface isopycnal slopes in height-coordinate and isopycnal-coordinate models, the boundary currents produced by both classes of models are very similar.

\section{Eddy shedding from the eastern boundary}

Individual eddies are tracked using the Okubo-Weiss parameter (Okubo 1970; Weiss 1991) at the surface and assigned to tracks based on the criteria set forth in Kurian et al. (2011). Eddies shed from the eastern boundary region propagate predominantly westward (Fig. 5). Most propagate faster than the theoretical midlatitude long Rossby wave speed, which is consistent with global observations of mesoscale eddy propagation (Chelton et al. 2011). Between $30^{\circ}$ and $40^{\circ} \mathrm{N}$, the number 
(a)

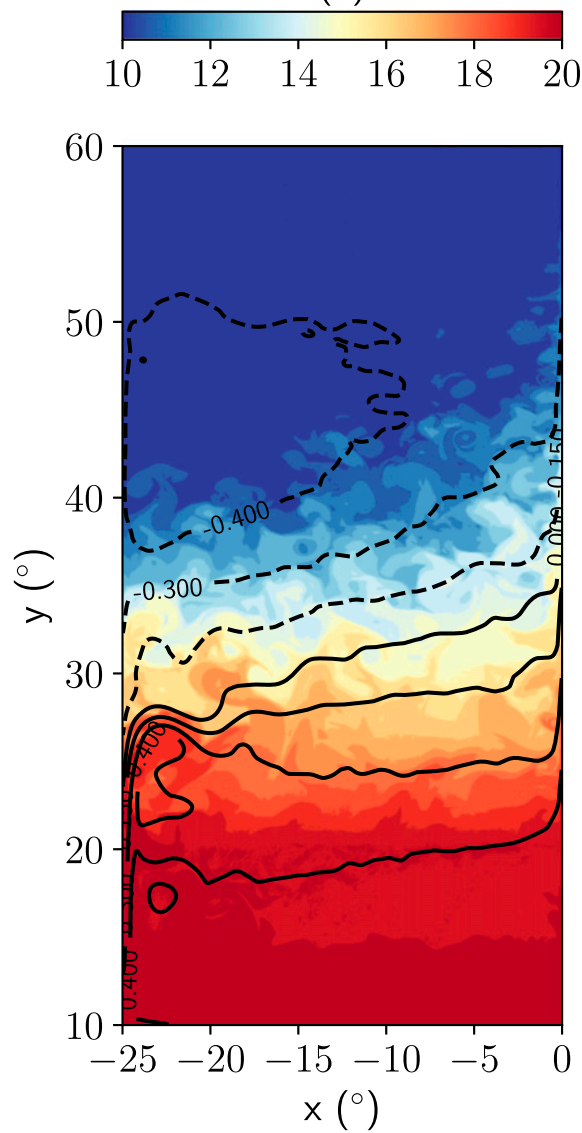

(b)
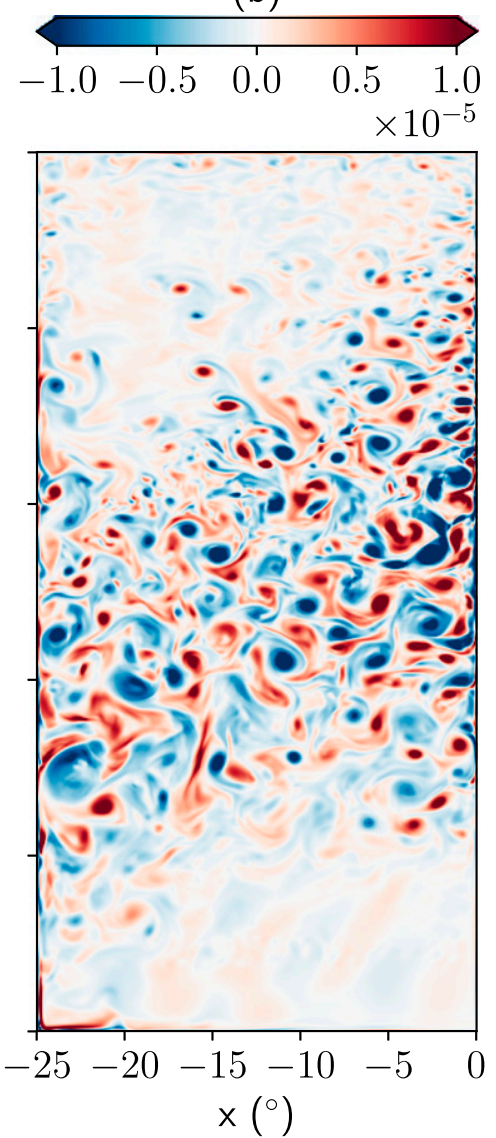

(c)
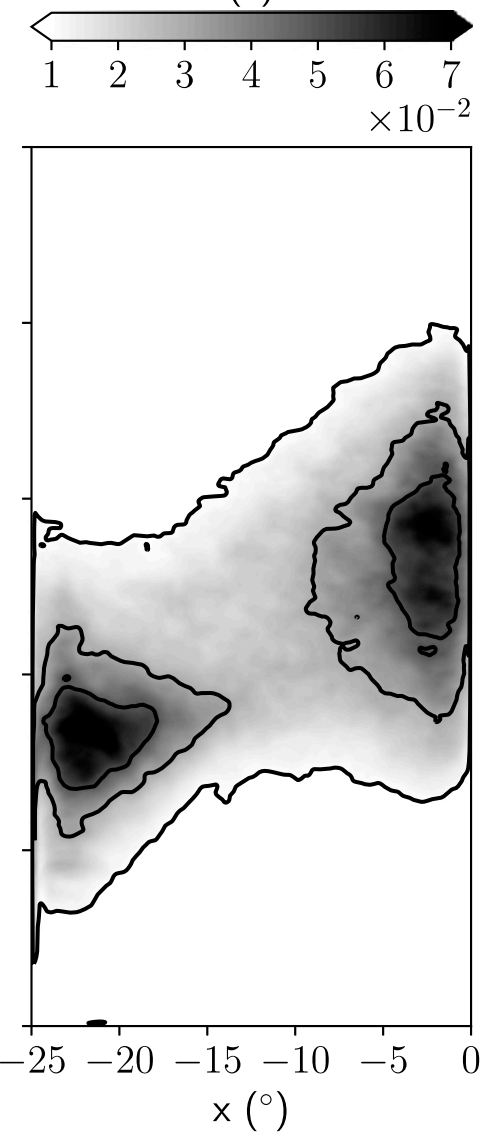

FIG. 4. (a) Instantaneous SST (shading; ${ }^{\circ} \mathrm{C}$ ) with mean SSH contours (m) overlaid. Contours are drawn at $-0.4,-0.3,-0.15,0,0.15,0.3$, and $0.4 \mathrm{~m}$. (b) Instantaneous vertically averaged vorticity $\left(\mathrm{s}^{-1}\right)$. (c) Vertically averaged EKE $\left(\mathrm{m}^{2} \mathrm{~s}^{-2}\right)$. Contours are drawn at $0.01,0.03$, and $0.05 \mathrm{~m}^{2} \mathrm{~s}^{-2}$.

of long-lived eddies seems to be smaller (Fig. 5). This is because these latitudes coincide with relatively strong onshore flow and eddies frequently interact with other eddies in this region. Since our tracking algorithm does not account for eddy mergers or bifurcations, very few eddies in this region appear to be long-lived. Figure $6 \mathrm{a}$ shows total number of tracks originating from east of $-2.5^{\circ}$, while Fig. $6 \mathrm{~b}$ shows the average duration of tracks originating east of $-2.5^{\circ}$. The former shows that there is a mild preference for anticyclonic eddies to be shed from the eastern boundary, while the latter shows that anticyclonic eddies outlast cyclonic eddies especially north of $30^{\circ} \mathrm{N}$. This preference is due to the nature of the EBC, which is warmer at the surface than water just offshore, so eddies originating from the EBC tend to have warm cores and are thus anticyclones. Thus, the eastern boundary current feeds warm water into these anticyclonic eddies, which then deposit it in the interior as they propagate offshore and dissipate, leading to the zonally asymmetric surface buoyancy distribution seen in Fig. 4a. This finding has been formalized in the next section by examining the terms of the buoyancy budget. We shall see that the overwhelming abundance of anticyclonic eddies has a significant impact on the residual circulation near the eastern boundary.

\section{Effect of eddies on circulation and heat transport near the eastern boundary}

The Eulerian picture of circulation near the eastern boundary is shown in Fig. 7. The eastward surface flow, driven by the baroclinic pressure gradient imposed at the surface, downwells and returns westward at around $1000 \mathrm{~m}$. The stretching and compressing of isopycnal layers at the surface and $1000 \mathrm{~m}$, respectively, support a vertically stacked structure of meridional velocity, with poleward flow at the surface and equatorward flow at $1000 \mathrm{~m}$.

In the presence of eddies the Eulerian picture of circulation differs considerably from the residual-mean picture (Fig. 8). Following Young (2012), we denote the isopycnal coordinate system by $(\tilde{x}, \tilde{y}, \tilde{b}, \tilde{t})$, for which the three components of residual velocity are 


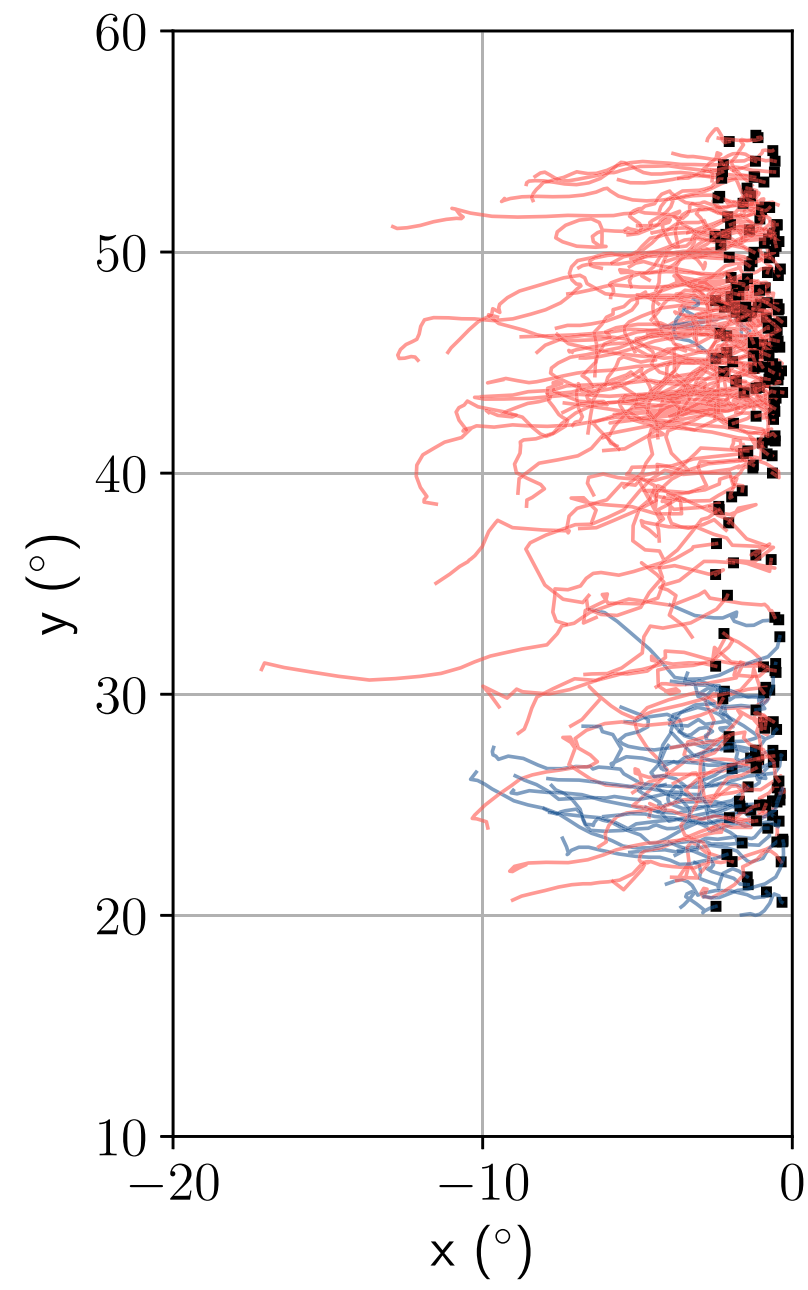

FIG. 5. Eddy tracks lasting at least 2 months originating from east of $-2.5^{\circ}$. Black squares indicate the location of origin of each track. Anticyclones (cyclones) are in red (blue).

$$
\begin{gathered}
\hat{u}=\frac{\overline{d e f}}{\bar{\sigma}}=\bar{u}+\frac{\overline{\sigma^{\prime} u^{\prime}}}{\bar{\sigma}}, \\
\hat{v}=\frac{\overline{\sigma e f}}{\bar{\sigma}}=\bar{v}+\frac{\overline{\sigma^{\prime} v^{\prime}}}{\bar{\sigma}}, \text { and } \\
w^{\sharp}=\bar{\zeta}_{\tilde{t}}^{\operatorname{def}}+\underbrace{\hat{u} \bar{\zeta}_{\tilde{x}}+\hat{v} \bar{\zeta}_{\tilde{y}}}_{\text {epipycnal }}+\underbrace{\hat{\bar{\sigma} \bar{\zeta}_{\tilde{b}}}}_{\text {diapycnal }},
\end{gathered}
$$

where $\bar{\sigma}=\bar{\zeta}_{\tilde{b}}$, and $\zeta$ is the isopycnal height. An overbar denotes averaging in time at constant buoyancy and a hat denotes the TWA of a quantity, defined as

$$
\hat{\theta} \stackrel{\text { def }}{=} \frac{\overline{\sigma \theta}}{\bar{\sigma}},
$$

for any field $\theta$. The TWA of any quantity can be decomposed into mean and eddy-induced components- these are first and second terms, respectively, on the RHS of (8) and (9). The eddy-induced component of the residual velocity is sometimes referred to as the "bolus" velocity. The superscript \# indicates a quantity that is not an explicit mean of any quantity, but appears in the TWA equations. The vertical velocity in the residual system is one such quantity, which is a combination of epipycnal (i.e., along isopycnals) and diapycnal components as indicated in (10). The term $\varpi$ represents diabatic forcing as an equivalent vertical velocity in isopycnal coordinates and is given by the material derivative of the buoyancy $b$; that is,

$$
\frac{D b}{D t}=\varpi
$$

Along the eastern boundary, the mean zonal velocity (Fig. 8a) is nearly identical to the Eulerian-mean zonal velocity. The zonal eddy-induced velocity (Fig. 8b) is opposite to the mean zonal velocity and sufficiently strong to reverse the residual velocity relative to the mean, so that the residual circulation is westward near the surface and eastward just below it. The westward residual velocity is the result of anticyclonic eddies physically transporting water warmer than the mean SST from the surface EBC toward the interior. The mean meridional velocity (Fig. 8d) shows the surface current stacked on top of a deeper undercurrent; the eddy-induced velocity (Fig. 8e) marginally opposes the meridional flow, and only very close to the surface. The residual meridional velocity (Fig. 8f) is thus nearly identical to the mean meridional velocity. The contribution of the epipycnal component (Fig. 8h) to the residual vertical velocity (Fig. 8i) is far greater than the diapycnal component (Fig. 8g). The residual vertical velocity shows that the EBC is associated with upwelling rather than downwelling seen in the Eulerian-mean velocity. This upwelling is predominantly a manifestation of zonal and meridional transport along sloping isopycnals.

TWA version of the buoyancy equation (12) written in height coordinates is

$$
b_{t}^{\sharp}+\hat{u} b_{x}^{\sharp}+\hat{v} b_{y}^{\#}+w^{\sharp} b_{z}^{\sharp}=\hat{\varpi},
$$

where $b^{\sharp}$ is the buoyancy mapped into height coordinates based on the mean position of the isopycnals. The steady state buoyancy budget is shown in Fig. 9. Note that the meridional and zonal velocities have been decomposed according to (8) and (9) to distinguish the mean and eddy components of buoyancy advection. Since $b_{x}^{\sharp}=-\bar{\zeta}_{\tilde{x}} \bar{\sigma}^{-1}, b_{y}^{\sharp}=-\bar{\zeta}_{\tilde{y}} \bar{\sigma}^{-1}$, and $b_{z}^{\sharp}=\bar{\sigma}^{-1}$, the terms in Figs. 9a, 9c, and 9f are $O\left(\bar{\sigma}^{-1}\right)$ and those in Figs. 9b, 9d, and 9e are $O\left(\bar{\sigma}^{-2}\right)$. Since $\bar{\sigma}$ is infinitesimally small in the layers squeezed at the surface, we are not confident of the numerical accuracy of the budget in the 
(a)

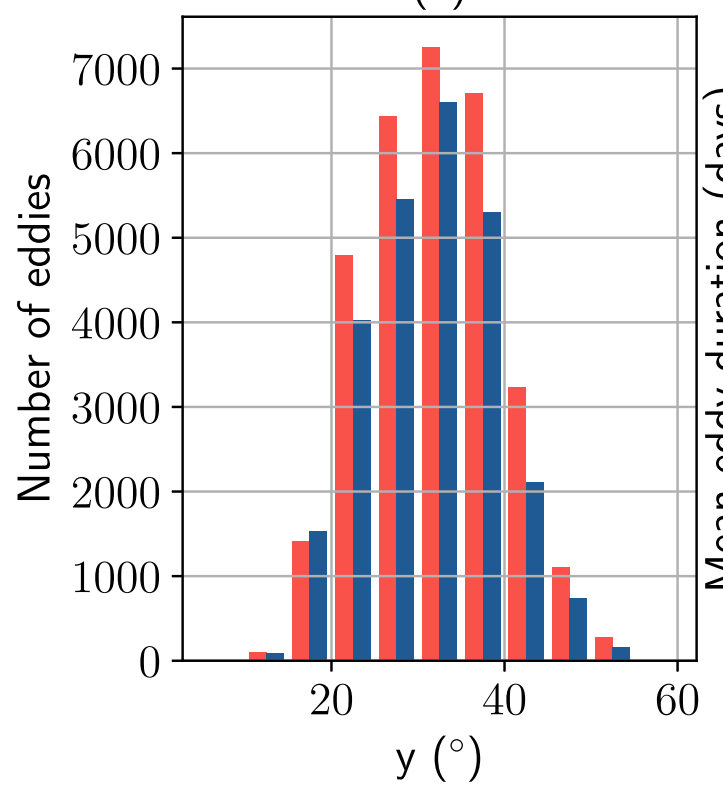

(b)

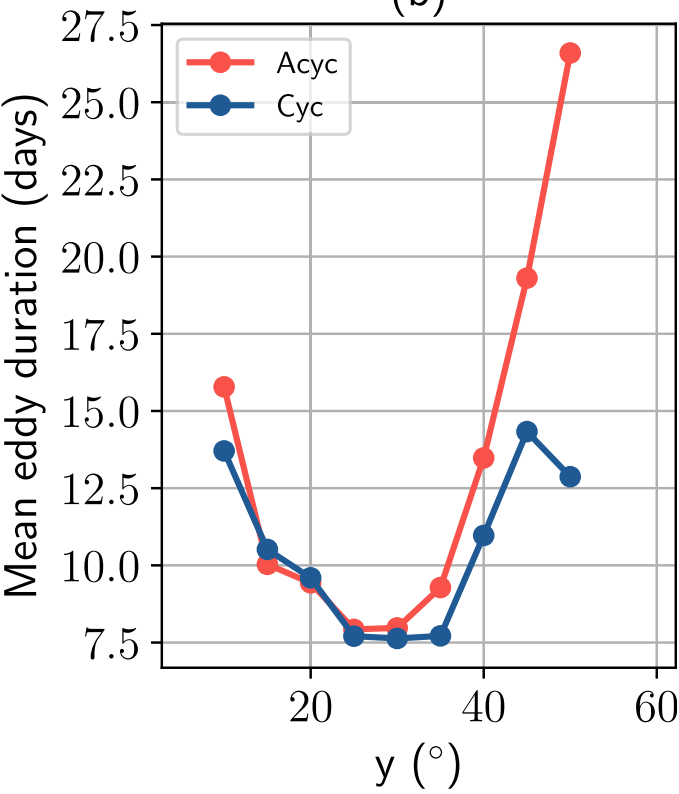

FIG. 6. (a) Total number of eddy tracks originating east of $-2.5^{\circ}$ from each latitude. (b) Average duration of eddy tracks originating east of $-2.5^{\circ}$ as a function of latitude. Anticyclones (cyclones) are in red (blue).

upper $\sim 20 \mathrm{~m}$. The following discussion is relevant to the budget below this depth as the values of $\bar{\sigma}$ become finite and the accuracy significantly increases.

The mean meridional velocity advects warm water northward along the eastern boundary (Fig. 9c), which is then transported offshore by the zonal eddy-induced velocity (Fig. 9b). These two factors contribute to the poleward tilt in SST along the eastern boundary seen in Fig. 4a. The primary balance in the surface current is between warming due to mean meridional advection and cooling due to vertical advection (Figs. 9c and 9e, respectively). This balance represents water flowing adiabatically northward and upward along sloping isopycnals. Additional contributions to the budget come from the zonal advection-which results in a net heating in the upper $300 \mathrm{~m}$ due to cancellation between the cooling mean flow and warming eddy flow (Figs. 9a and 9b, respectively) - and weak cooling by eddy meridional advection and surface diabatic forcing (Figs. 9d and 9f, respectively). The buoyancy budget in the undercurrent is the opposite of the surface current, except for the absence of eddy meridional advection and surface cooling. Since eddies do not play a significant role in meridional advection of buoyancy (Fig. 9d), coarseresolution models are able to produce the tilt in SST as well (e.g., Schloesser et al. 2012). In the absence of eddies, the zonal transport of buoyancy is achieved through horizontal diffusion.

\section{Momentum and thickness budgets near the eastern boundary}

Here we inspect the budgets of momentum and thickness near the eastern boundary to unravel the
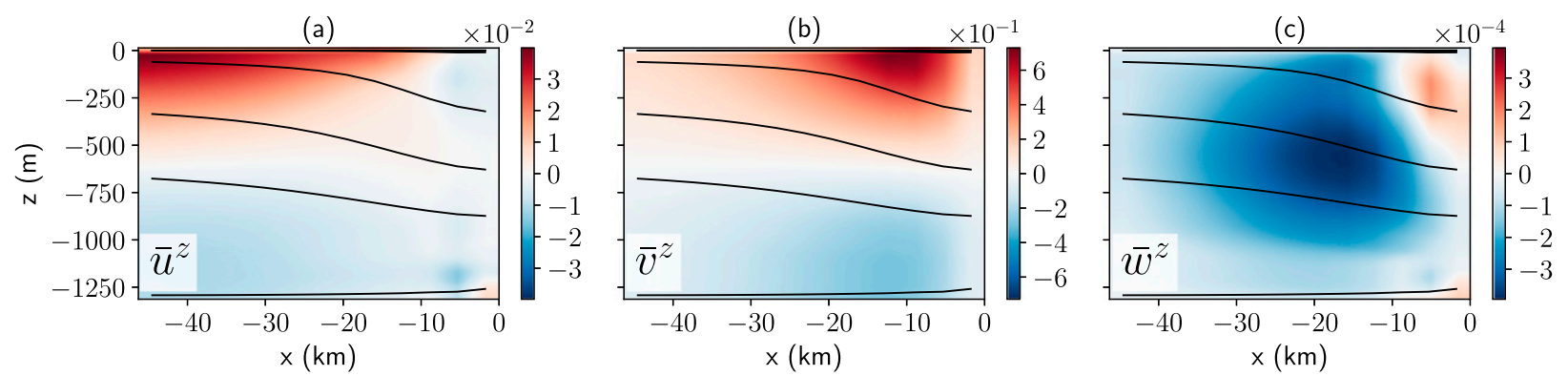

FIG. 7. Zonal section of mean velocity $\left(\mathrm{m} \mathrm{s}^{-1}\right)$, meridionally averaged between $35^{\circ}$ and $37^{\circ} \mathrm{N}$ near the eastern boundary. The black contours represent mean positions of isopycnals at intervals of $0.003 \mathrm{~m} \mathrm{~s}^{-2}$. 
(a)

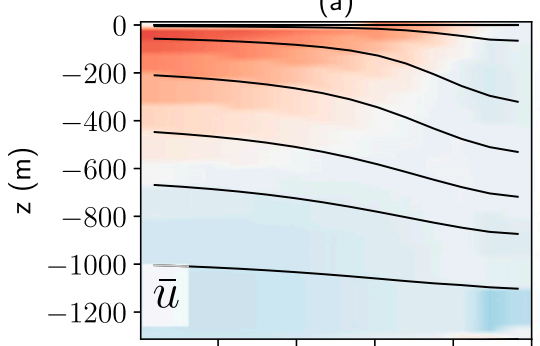

(d)

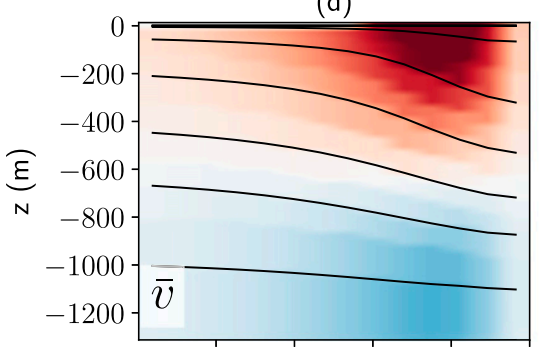

(g)

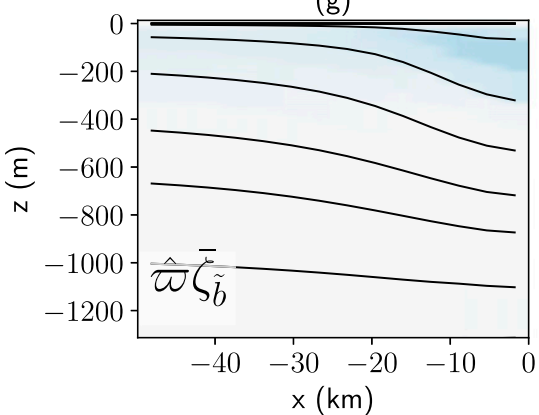

(b)

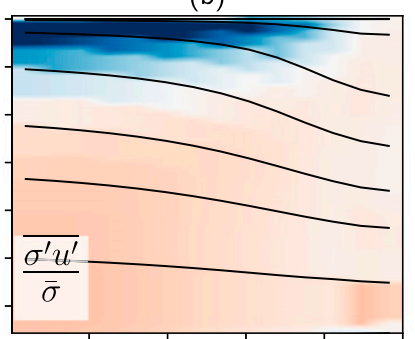

(e)

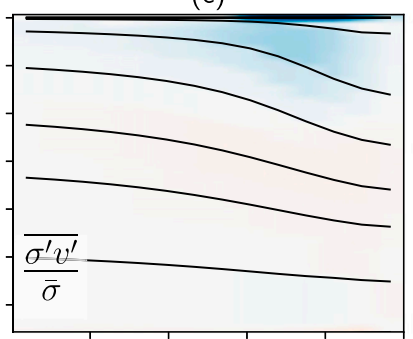

(h)

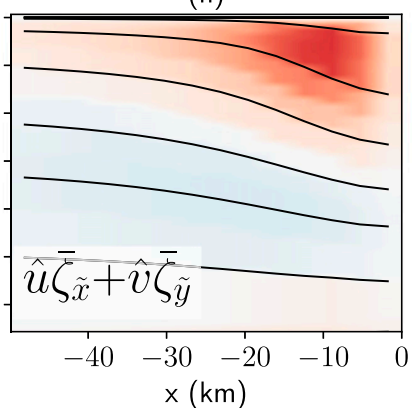

(c)

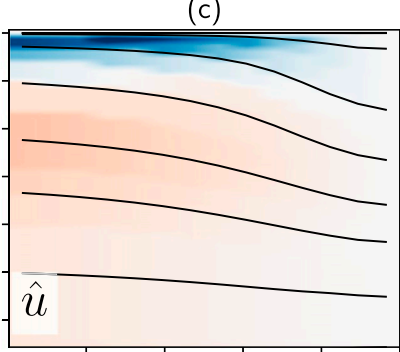

(f)

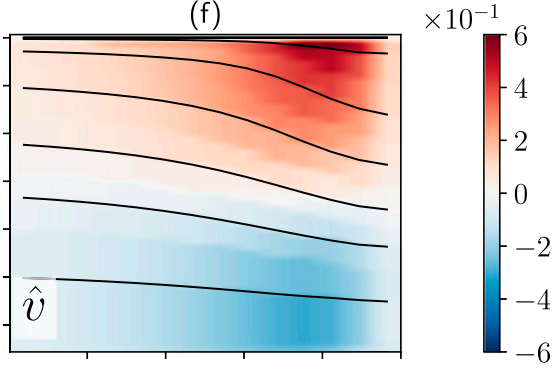

(i)

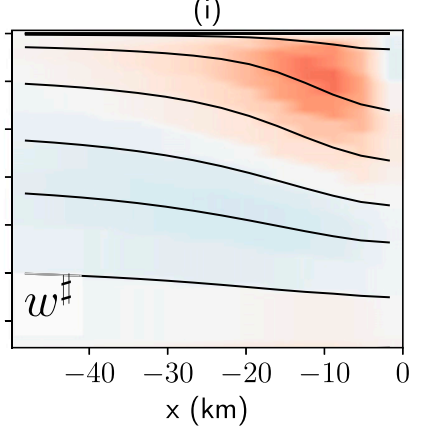

$\times 10^{-2}$

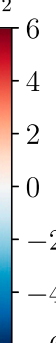
$\times 10^{-4}$

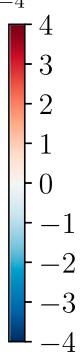

FIG. 8. Zonal section of mean TWA velocity $\left(\mathrm{m} \mathrm{s}^{-1}\right)$, averaged between $36^{\circ}$ and $37^{\circ} \mathrm{N}$, near the eastern boundary. Given are the residual (a)-(c) zonal, (d)-(f) meridional, and (g)-(i) vertical velocities and their respective components. For the horizontal velocities, the columns give the (left) time mean velocities at constant buoyancy, (center) the eddy-induced velocities, and (right) the residual velocities. The residual vertical velocity in the right column is divided into epipycnal (in the center column) and diapycnal (in the left column) components. See (8)-(10). The black contours represent mean positions of isopycnals at intervals of $0.002 \mathrm{~m} \mathrm{~s}^{-2}$.

dominant physical processes that drive the eastern boundary current and maintain its cross-shore structure.

The zonal momentum balance near the boundary (not shown) is geostrophic to a high degree of accuracy; that is,

$$
f \hat{v}=\bar{m}_{\tilde{x}},
$$

where $\bar{m}$ is the mean Montgomery potential. This balance has been found by a number of previous studies (McCreary et al. 1986; Cessi and Wolfe 2009; Furue et al. 2013; Connolly et al. 2014; Cessi and Wolfe 2013; Benthuysen et al. 2014).

Retaining only the dominant terms, the meridional momentum equation is

$$
f \hat{u}=-\bar{m}_{\tilde{y}}+\hat{Y}-\nabla \cdot \mathbf{E}^{v},
$$

where $\hat{Y}$ represents the TWA nonconservative terms (lateral friction in this case) and the EP flux vector is given by

$$
\mathbf{E}^{v} \stackrel{\operatorname{def}}{=}\left(\widehat{u^{\prime \prime} v^{\prime \prime}}\right) \overline{\mathbf{e}}_{1}+\left(\overline{\frac{\zeta^{\prime 2}}{2 \bar{\sigma}}}\right) \overline{\mathbf{e}}_{2}+\left(\overline{\frac{\zeta^{\prime} m_{\tilde{y}}^{\prime}}{\bar{\sigma}}}\right) \overline{\mathbf{e}}_{3},
$$

where the basis vectors are

$$
\overline{\mathbf{e}}_{1} \stackrel{\text { def }}{=} \mathbf{i}+\bar{\zeta}_{x} \mathbf{k}, \quad \overline{\mathbf{e}}_{2} \stackrel{\text { def }}{=} \mathbf{j}+\bar{\zeta}_{x} \mathbf{k}, \quad \overline{\mathbf{e}}_{3} \stackrel{\text { def }}{=} \bar{\sigma} \mathbf{k},
$$

and deviations from mean quantities are defined as

$u^{\prime \prime}=u-\hat{u}, \quad v^{\prime \prime}=v-\hat{v}, \quad \zeta^{\prime}=\zeta-\bar{\zeta}, \quad m^{\prime}=m-\bar{m}$.

The first term in RHS of (16) is a Reynolds stress term that acts in the zonal direction along isopycnals. The third term, acting in the vertical direction, is the form drag, which arises from the correlation between fluctuations of isopycnal height and the gradient of the Montgomery potential. The middle term, acting in the 
(a)

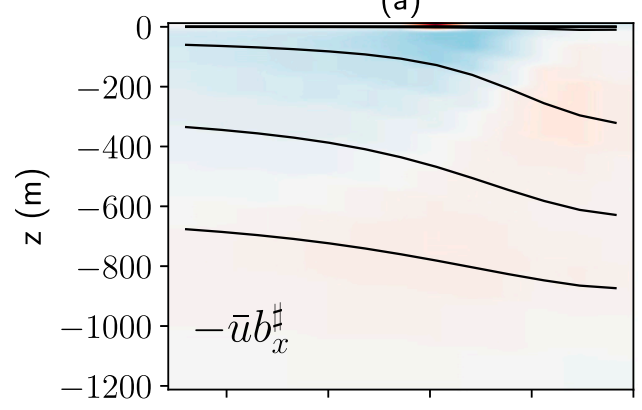

(c)

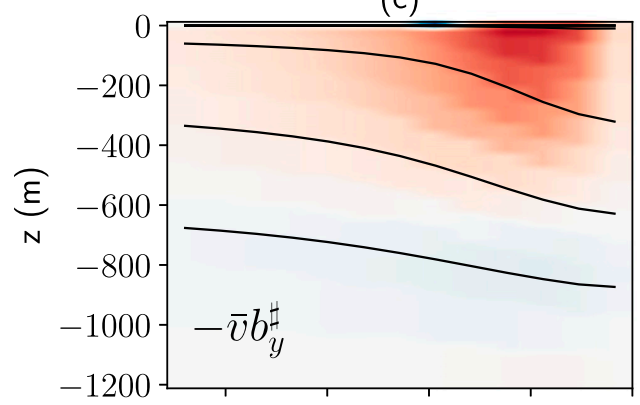

(e)

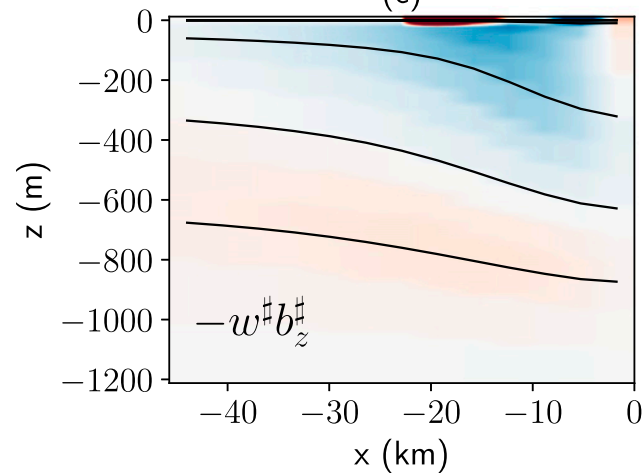

(b)

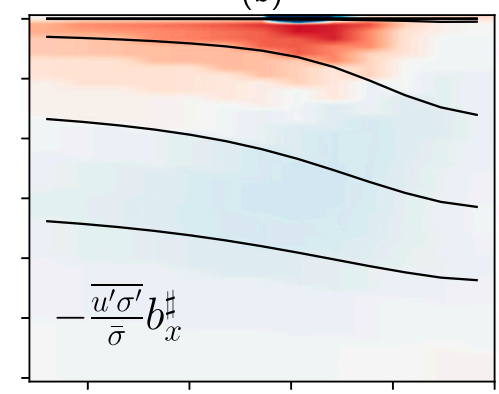

(d)

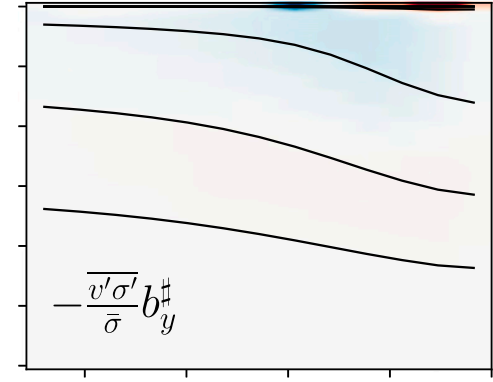

(f)

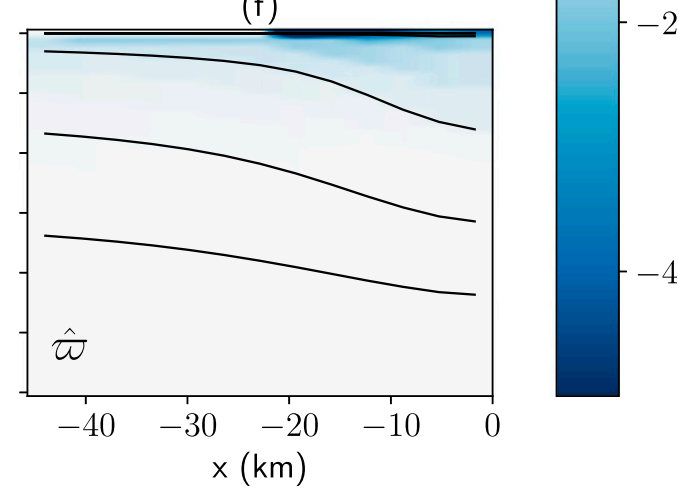

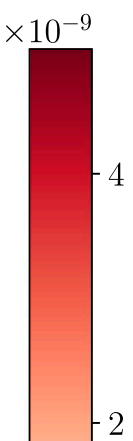

$-0$

$-2$

FIG. 9. Zonal section of the terms of the residual-mean buoyancy budget from (13), averaged between $35^{\circ}$ and $37^{\circ} \mathrm{N}$, near the eastern boundary $\left(\mathrm{m} \mathrm{s}^{-3}\right)$. Advection by (a) zonal mean and (b) eddy-induced velocities is shown, as well as that by (c) meridional mean and (d) eddy-induced velocities. (e) Advection by vertical velocity and (f) tendency by diabatic transformation are also shown. The black contours represent mean positions of isopycnals at intervals of $0.003 \mathrm{~m} \mathrm{~s}^{-2}$.

meridional direction along isopycnals, is negligibly small but is retained to maintain the correspondence of the divergence of the EP flux with the eddy pressure forcing (see Young 2012; Maddison and Marshall 2013). The divergence for any vector $\mathbf{q}=l \overline{\mathbf{e}}_{1}+m \overline{\mathbf{e}}_{2}+n \overline{\mathbf{e}}_{3}$ is given by $\nabla \cdot \mathbf{q}=\bar{\sigma}^{-1}(\bar{\sigma} l)_{\tilde{x}}+\bar{\sigma}^{-1}(\bar{\sigma} m)_{\tilde{y}}+\bar{\sigma}^{-1}(\bar{\sigma} n)_{\tilde{b}}$, so that

$$
\nabla \cdot \mathbf{E}^{v}=\frac{\left(\bar{\sigma} \widehat{u^{\prime \prime} v^{\prime \prime}}\right)_{\tilde{x}}}{\bar{\sigma}}+\frac{\left(\overline{\zeta^{\prime 2}}\right)_{\tilde{y}}}{2 \bar{\sigma}}+\frac{\left(\overline{\zeta^{\prime} m_{\tilde{y}}^{\prime}}\right)_{\tilde{b}}}{\bar{\sigma}}
$$

\section{(Young 2012).}

Very close to the boundary, the gradient of Montgomery potential (Fig. 10b) is balanced by the horizontal friction (Fig. 10d). Elsewhere, the primary balance in the meridional momentum equation (15) is between the Coriolis acceleration (Fig. 10a), gradient of Montgomery potential, and the EP flux divergence (Fig. 10c). Both the Coriolis force and the gradient of the Montgomery potential tend to accelerate the meridional flow and are countered by EP flux divergence. The EP flux divergence in (19) is dominated by the zonal Reynolds stress (Fig. 11a) and the form drag (Fig. 11b). The Reynolds stress is directed away from (toward) the eastern boundary above (below) $500 \mathrm{~m}$, while the form drag transports momentum from the surface to the deeper levels. The overall role of the eddy momentum 
(a)

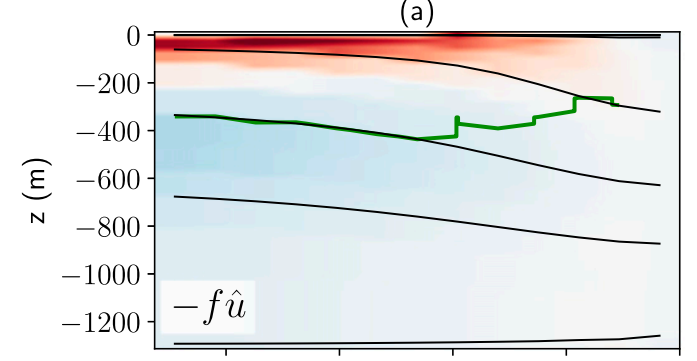

(c)

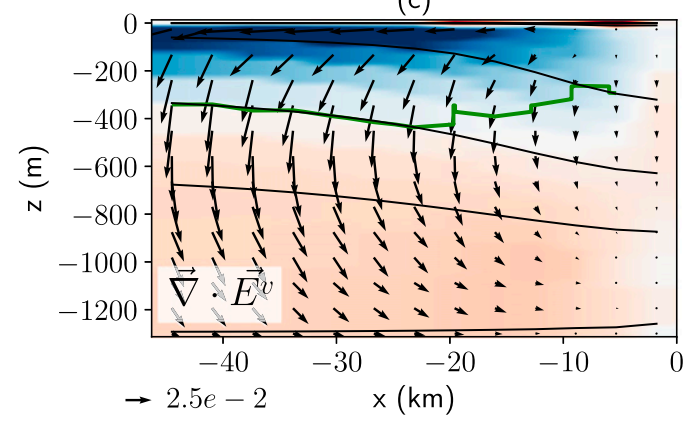

(b)

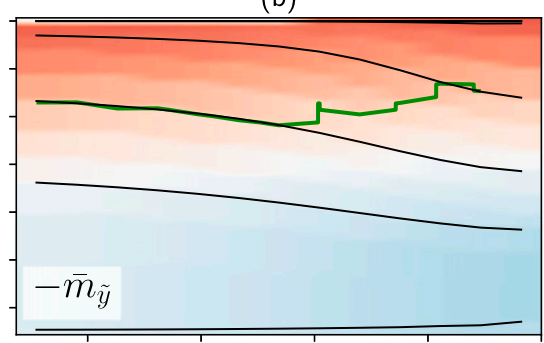

(d)

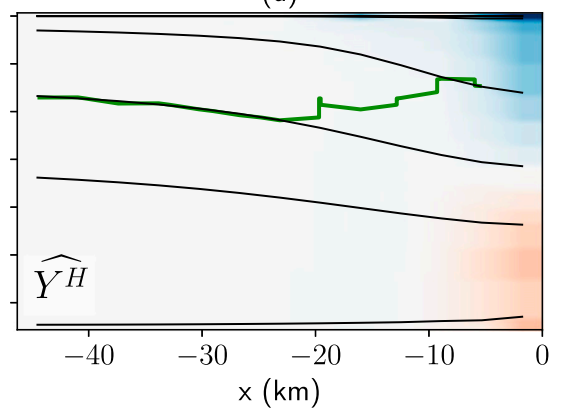

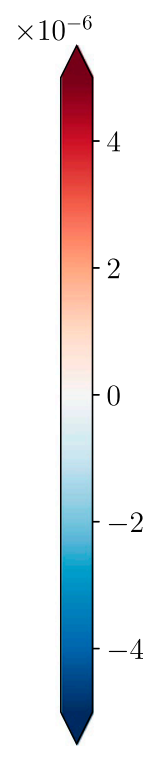

2

(1)

$-2$

$-4$

FIG. 10. Zonal section of the dominant components of meridional TWA momentum budget $\left(\mathrm{m} \mathrm{s}^{-2}\right)$ from (15), namely, (a) the Coriolis acceleration, (b) the gradient of Montgomery potential, (c) the divergence of EP flux, and (d) the horizontal friction, averaged between $35^{\circ}$ and $37^{\circ} \mathrm{N}$, near the eastern boundary. The green line is the bottom boundary of the swash zone. Arrows indicate the direction of the EP fluxes in the $x-z$ plane. The black contours represent mean positions of isopycnals at intervals of $0.003 \mathrm{~m} \mathrm{~s}^{-2}$.

fluxes is to transport momentum from the surface current to the undercurrent. Since the currents are oppositely directed, this results in a slowing of both. Note, however, that the vertical transfer of momentum is not direct; instead, the EP flux emanates westward from the surface current, then turns downward at about a Rossby deformation radius from the eastern boundary, and then feeds eastward into the undercurrent. The downward flux of momentum is caused by the interfacial form drag resulting from the eddies shed from the eastern boundary. In the interior, the form drag homogenizes the momentum in the vertical direction, thereby ensuring that the EBCs are confined near the boundary. The eddies are themselves roughly a Rossby radius in size, so they can only form when they are at least a Rossby radius away from the eastern boundary. In the absence of form drag, the Montgomery potential gradient would accelerate the seaward flank of the EBC, resulting in a broader but slower current. The influence of form drag becomes small close to the coast, and it is this distribution of the form drag that sets the width of the EBC, as we show in section 8.

Further insights can be gained by forming a thickness budget similar to CW13. The thickness equation is given by
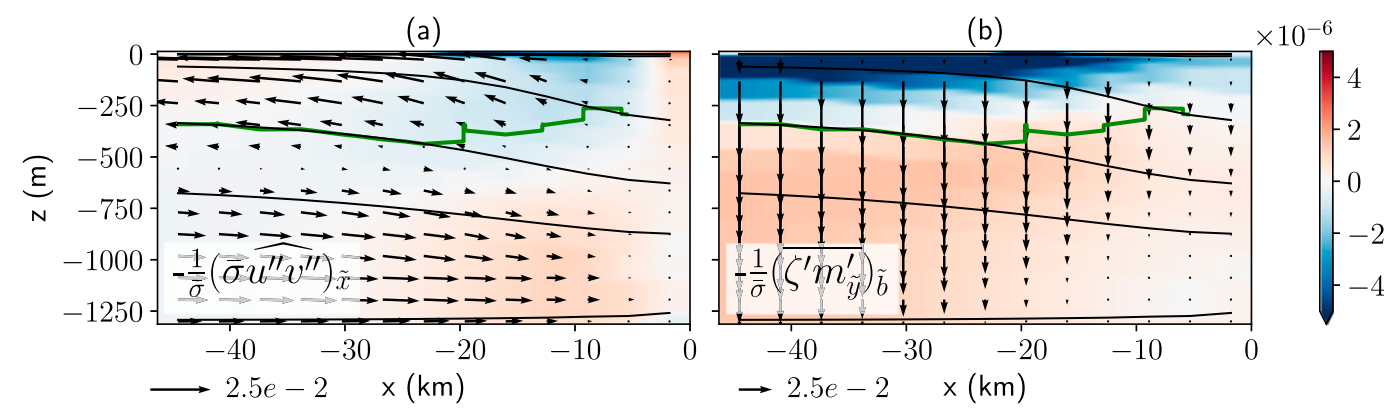

FIG. 11. Zonal section of dominant components of meridional EP flux divergence $\left(\mathrm{m} \mathrm{s}^{-2}\right.$ ) from (19), namely, (a) divergence of the zonal Reynolds stress and (b) form drag, averaged between $35^{\circ}$ and $37^{\circ} \mathrm{N}$, near the eastern boundary. The green line is the bottom boundary of the swash zone. Arrows indicate the direction of the fluxes from (16). The black contours represent mean positions of isopycnals at intervals of $0.003 \mathrm{~m} \mathrm{~s}^{-2}$. 
(a)

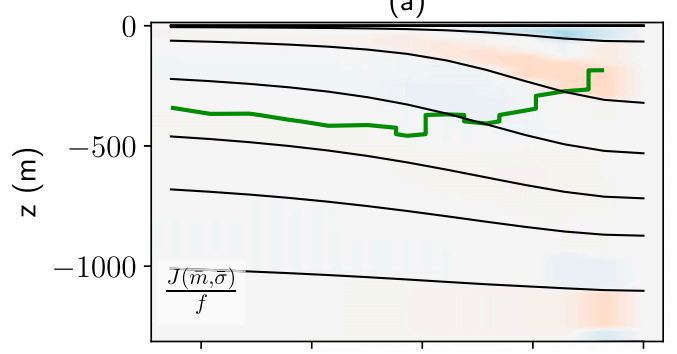

(c)

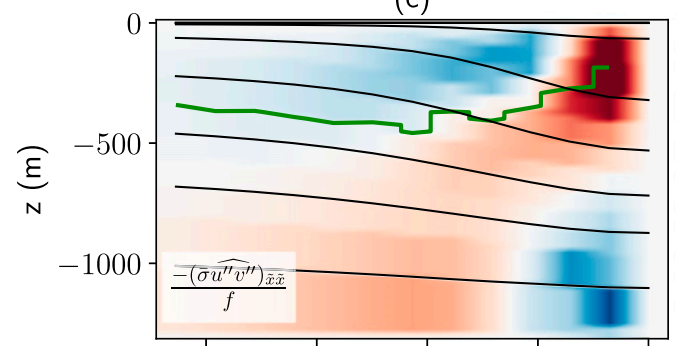

(e)

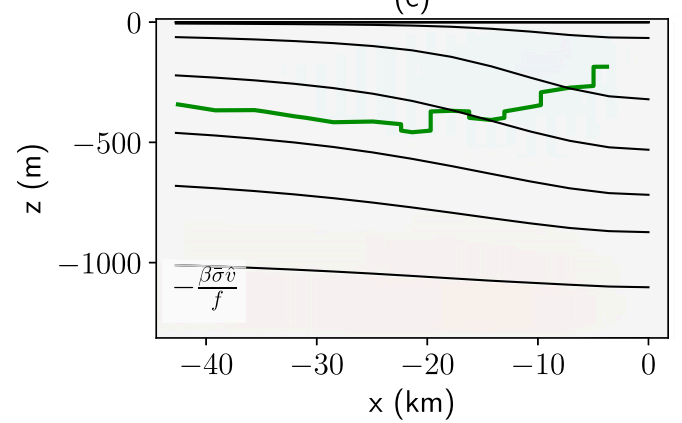

(b)

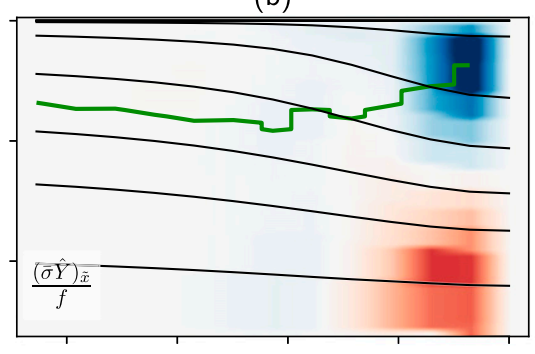

(d)

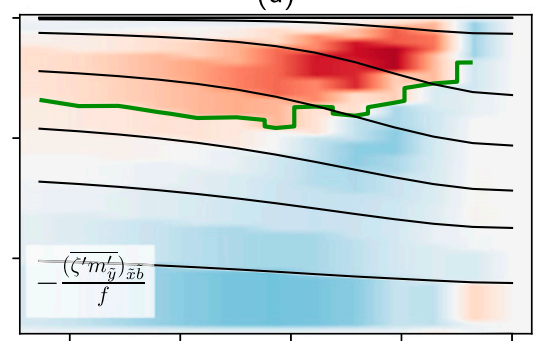

(f)
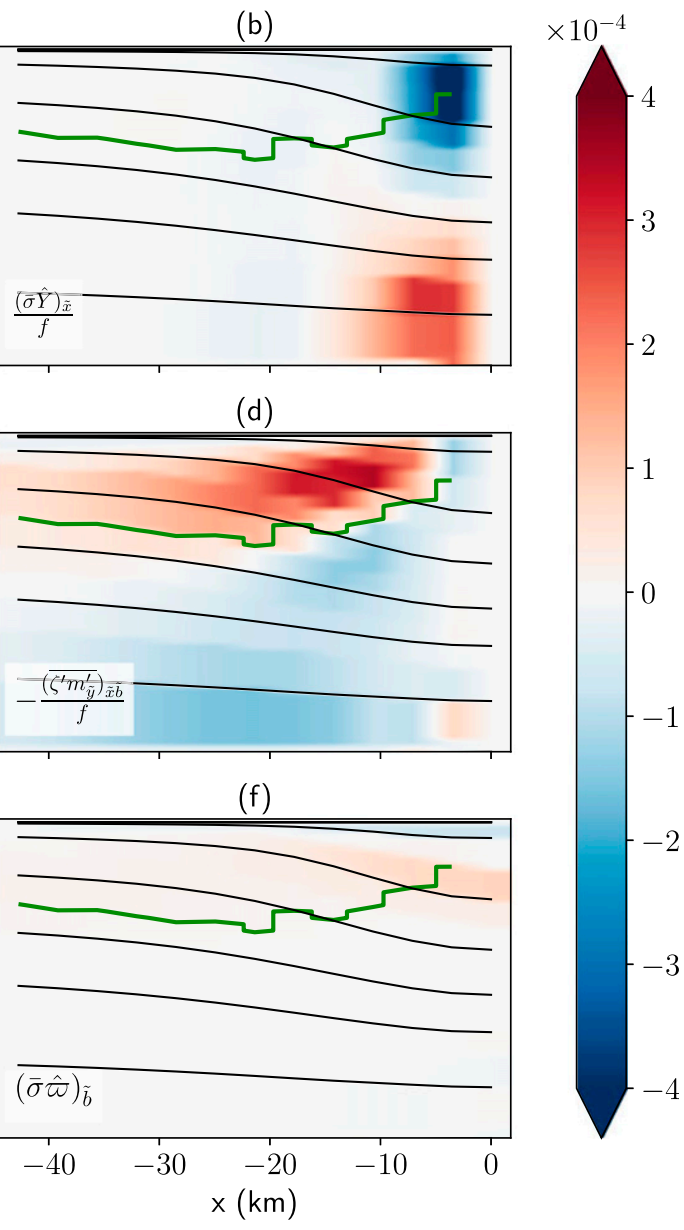

1

0

$-1$

$-2$

$-3$
FIG. 12. Zonal sections of the terms of the thickness budget in (21), averaged between $35^{\circ}$ and $37^{\circ} \mathrm{N}$, near the eastern boundary. (a),(e) Advection due to geostrophic velocity, (b) lateral friction, (c),(d) components of the EP fluxes, and (f) the diapycnal component. The black contours represent mean positions of isopycnals at intervals of $0.002 \mathrm{~m} \mathrm{~s}^{-2}$.

$$
(\bar{\sigma} \hat{u})_{\tilde{x}}+(\bar{\sigma} \hat{v})_{\tilde{y}}+(\bar{\sigma} \hat{\varpi})_{\tilde{b}}=0 .
$$

Multiplying (14) and (15) by $\bar{\sigma}$ and eliminating $\bar{\sigma} \hat{u}$ and $\bar{\sigma} \hat{v}$ using (20) gives

$$
\begin{aligned}
& \frac{1}{f}\left[\bar{m}_{\tilde{x}} \bar{\sigma}_{\tilde{y}}-\bar{m}_{\tilde{y}} \bar{\sigma}_{\tilde{x}}-\beta \bar{\sigma} \hat{v}+(\bar{\sigma} \hat{Y})_{\tilde{x}}-\left(\bar{\sigma} \nabla \cdot \mathbf{E}^{v}\right)_{\tilde{x}}\right] \\
& +(\bar{\sigma} \hat{\omega})_{\tilde{b}}=0
\end{aligned}
$$

which is the balance explored by CW13. We obtain a similar balance as shown in Fig. 12. As with their study, both the diabatic term and the $\beta$ effect have negligible impacts on the EBC. In addition, the first two terms of (21), representing advection of thickness by the geostrophic flow, cancel each other so that the dominant balance is between the remaining terms. That is,

$$
(\bar{\sigma} \hat{Y})_{\tilde{x}}-\left(\bar{\sigma} \nabla \cdot \mathbf{E}^{v}\right)_{\tilde{x}}=0
$$

The viscous stress term $\bar{\sigma} \hat{Y}$ is important only very near the boundary, so the physics that explains the zonal structure of EBC is concealed in the divergence of the EP flux. In the next section we devise parameterizations for the components of EP flux divergence that help illuminate their role in determining the zonal structure of the EBC.

\section{Parameterization of Eliassen-Palm flux}

We start by noting the similarity in the spatial structures of form drag (Fig. 11b) and eddy-induced velocity (Fig. 8b). This close correspondence is a direct consequence of the form of the EP flux divergence appearing in the meridional momentum equation. Using the hydrostatic relation, $m_{\tilde{b}}+\zeta=0$, and the definition of thickness, $\sigma=\zeta_{\tilde{b}}$, the last two terms in (19) can be simplified as 


$$
\frac{\left(\overline{\zeta^{\prime 2}}\right)_{\tilde{y}}}{2 \bar{\sigma}}+\frac{\left(\overline{\zeta^{\prime} m_{\tilde{y}}^{\prime}}\right)_{\tilde{b}}}{\bar{\sigma}}=\frac{\overline{\zeta^{\prime} \zeta_{\tilde{y}}^{\prime}}+\overline{\zeta_{\tilde{b}}^{\prime} m_{\tilde{y}}^{\prime}}+\overline{\zeta^{\prime} m_{\tilde{b} \tilde{y}}^{\prime}}}{\bar{\sigma}}=\frac{\overline{\sigma^{\prime} m_{\tilde{y}}^{\prime}}}{\bar{\sigma}}
$$

Assuming eddies to be geostrophic, we have

$$
f u^{\prime}=-m_{\tilde{y}}^{\prime},
$$

so

$$
\frac{\left(\overline{\zeta^{\prime} m_{\tilde{y}}^{\prime}}\right)_{\tilde{b}}}{\bar{\sigma}}+\frac{\overline{\zeta_{\tilde{y}}^{\prime 2}}}{2 \sigma}=-f \frac{\overline{\sigma^{\prime} u^{\prime}}}{\bar{\sigma}} .
$$

The second term on the LHS of (25) is negligible in this case, so the eddy-induced velocity is approximately proportional to the form drag. This similarity between the eddy-induced velocity and the form drag has been noted in previous studies (e.g., Greatbatch 1998).

Assuming a flux-gradient relationship between the eddy thickness flux and the mean thickness gradient gives a parameterization of the form drag (e.g., Greatbatch and Lamb 1990; Gent et al. 1995; Greatbatch 1998; Ferreira and Marshall 2006; Saenz et al. 2015):

$$
-\left(\overline{\zeta^{\prime} m_{\tilde{y}}^{\prime}}\right)_{\tilde{b}} \simeq f \overline{\sigma^{\prime} u^{\prime}}=-K_{e} f \bar{\sigma}_{x} .
$$

Applying this form drag parameterization to the present case requires a spatially varying eddy diffusivity $K_{e}$, since the maximum in the form drag is located offshore of the maximum thickness gradient. To determine the zonal variation of $K_{e}$, the zonal domain is split into $0.1^{\circ}$ bins and, for each bin, values of the predictor $\bar{\sigma}_{\tilde{x}}$ and predictand $-\left(\overline{\zeta^{\prime} m_{\tilde{y}}^{\prime}}\right)_{\tilde{b}}$ are sampled for all layers thicker than $1 \mathrm{~mm}$ at all grid points within $1^{\circ}$ in the meridional direction on either side of the latitude of interest. The value of $K_{e}$ for each bin is then estimated as the negative of regression slope divided by $f$. The data within each bin and the corresponding regression lines for $36^{\circ} \mathrm{N}$ are shown in Fig. 13a. The eddy diffusivity increases dramatically as one moves away from the boundary.

There are several reasons to expect the diffusivity to be smaller near the boundary than in the interior. The first reason is purely geometrical and based on mixing length arguments: since the mixing length cannot be larger than the distance to the boundary, the diffusivity must decrease as the boundary is approached. The second reason is that strong mean flows tend to suppress mixing in the cross-stream direction (e.g., Ferrari and Nikurashin 2010; Klocker et al. 2012; Klocker and Abernathey 2014), so the mere presence of the EBC should suppress cross-shore mixing. The expected profile of eddy diffusivity, from Ferrari and Nikurashin (2010), has the form

$$
K_{e}=\frac{a}{1+b\left(c_{w}-\bar{v}\right)^{2}} \mathrm{EKE},
$$

where $a$ and $b$ are parameters related to time and space scales of the eddies, and $c_{w}$ is the along-stream eddy speed Bates et al. (2014). In our case we assume $c_{w} \approx 0$. This curve indeed gives a reasonable representation of $K_{e}$ for $a \sim 3.2 \times 10^{6} \mathrm{~s}$ and $b \sim 2 \times 10^{4} \mathrm{~m}^{-2} \mathrm{~s}^{2}$ (Fig. 13b). Zonal profiles of $\bar{v}^{2}$ and EKE were obtained by averaging vertically. ${ }^{1}$

A detailed examination of diffusivity suppression by mean flows is beyond the scope of this study, so we proceed with a simple empirical model of the variation of $K_{e}$ :

$$
K_{e}=\frac{K_{e, I}}{2}\left[1-\tanh \left(\frac{x+L_{1}}{L_{2}}\right)\right],
$$

where $K_{e, I}$ is the interior value (Fig. 13b). This curve is found suitable at a number of other meridional locations, although the parameters $K_{e, I}, L_{1}$, and $L_{2}$ vary with latitude (Fig. 14). Both length scales, $L_{1}$ and $L_{2}$, decrease with latitude at a rate faster than the variation of the deformation radius (Fig. 14b) - the processes responsible for setting these length scales are currently unknown and are a subject of further study.

Reynolds stress divergence can be parameterized as either a linear drag on meridional velocity (following CW13),

$$
-\frac{\left(\bar{\sigma} \widehat{u^{\prime \prime} v^{\prime \prime}}\right)_{\tilde{x}}}{\bar{\sigma}}=r \hat{v}
$$

where $r$ is the drag coefficient, or as a diffusion of momentum (Fig. 15c),

$$
-\frac{\left(\bar{\sigma} \widehat{u^{\prime \prime} v^{\prime \prime}}\right)_{\tilde{x}}}{\bar{\sigma}}=\frac{\left(A_{h} \bar{\sigma} \hat{v}_{\tilde{x}}\right)_{\tilde{x}}}{\bar{\sigma}}
$$

where $A_{h}$ is the viscosity. For small slopes, $\bar{\sigma}$ can be passed through the derivative, so that

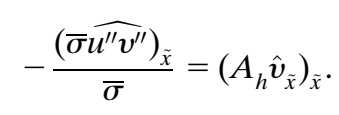

Figure 15 indicates that both parameterizations represent the vertical structure of the Reynolds stress divergence reasonably well but struggle to produce the correct horizontal distribution. In particular, linear drag and momentum diffusion produce features that are too broad and two narrow, respectively. The values of the drag coefficient and eddy viscosity are estimated in a

\footnotetext{
${ }^{1}$ The velocity is squared then averaged vertically; since the flow is baroclinic, averaging then squaring would give zero.
} 
(a)

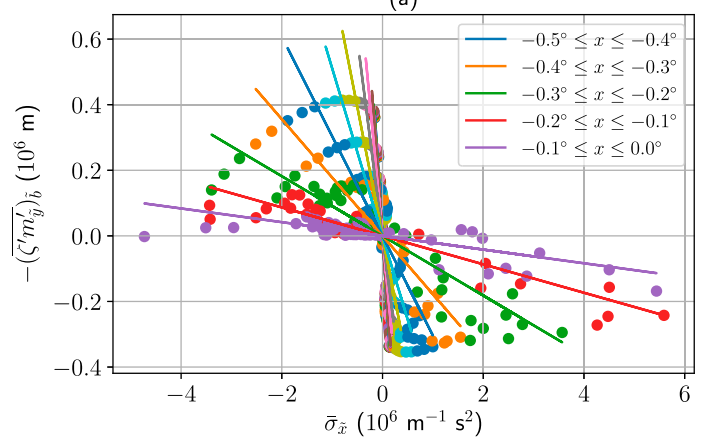

(b)

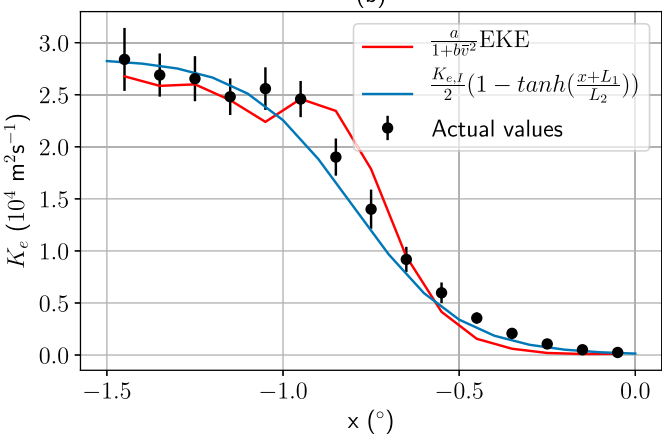

FIG. 13. (a) Lines of regression of $-\left(\overline{\zeta^{\prime} m_{\tilde{y}}^{\prime}}\right)_{\tilde{b}}$ on $\bar{\sigma}_{\tilde{x}}$ in zonal bins of $0.1^{\circ}$ between $35^{\circ}$ and $37^{\circ} \mathrm{N}$. The data used for each bin are shown by filled circles. Bins west of $-0.5^{\circ}$ are omitted from the legend for clarity. (b) The estimated value of $K_{e}$ as a function of distance from the eastern boundary is shown by filled black circles. The value of $K_{e}$ for any given bin is $-s / f$, where $s$ is the slope of the lines in (a). Error bars represent \pm 3 standard deviations. Red and blue curves represent fits obtained from (27) and (28), respectively.

similar manner as the eddy diffusivity, although all points within $0.5^{\circ}$ of the eastern boundary are treated equivalently so as to obtain a single value of $r$ or $A_{h}$. It is found that $r \sim 10^{-6} \mathrm{~s}^{-1}$ and $A_{h} \sim 200 \mathrm{~m}^{2} \mathrm{~s}^{-1}$.

We use these parameterizations in the next section to illustrate the physics responsible for determining the zonal structure of the EBC.

\section{A model of the eastern boundary current}

The essential physics of the eastern boundary currents can be understood using a two-layer analog of the TWA equations linearized about a resting thickness profile. The two layers are intended to represent the poleward surface current and the equatorward undercurrent.

The two layers are separated by an interface whose elevation above its rest level is $\eta(x, y)$. The model is formulated on the $\beta$ plane. The resting thicknesses of the two layers are taken to be equal so $H_{1}=H_{2}=H / 2$, where $H=1500 \mathrm{~m}$ is the total depth of the fluid (here taken to be the depth range of the surface and undercurrents shown in Fig. 8). The flow is driven by buoyancy forcing, so in the absence of bottom topography or significant bottom drag, the circulation is purely baroclinic. The equations of motion are

$$
\begin{aligned}
-f \hat{v} & =g^{\prime} \bar{\eta}_{x}, \\
f \hat{u} & =g^{\prime} \bar{\eta}_{y}+\frac{4 f K_{e}}{H} \bar{\eta}_{x}+A \nabla^{2} \hat{v}-r \hat{v}, \quad \text { and } \\
\nabla \cdot \hat{\mathbf{u}} & =\frac{4}{H} \bar{w}_{i},
\end{aligned}
$$

where $K_{e}$ is given by (28). See the appendix for a derivation. The meridional velocity is assumed to be in geostrophic balance. This assumption holds very well near the eastern boundary and is consistent with previous studies (e.g., McCreary et al. 1986; Furue et al. 2013). Inclusion of the form drag term in the zonal momentum equation leads to a smoother zonal velocity profile in the interior but otherwise does not significantly affect the solution. All the necessary dynamics to show that a zonally variable diffusivity produces a narrow eastern boundary current are captured in this model.

Following Pedlosky and Spall (2005), we set the interfacial velocity as a relaxation to a meridional profile $\theta$ with an inverse time-scale $\gamma$ :

$$
\bar{w}_{i}=\gamma(\bar{\eta}-\theta),
$$

where

$$
\theta= \begin{cases}\eta_{s} & y<y_{S}, \\ \eta_{s}+s\left(y-y_{S}\right) & y_{S} \leq y \leq y_{N}, \\ \eta_{s}+s\left(y_{N}-y_{S}\right) & y>y_{N},\end{cases}
$$

where $s$ is the slope.

The system in (32)-(34) supports a narrow boundary layer of width

$$
\delta=\sqrt{\frac{A}{K_{e}}} L_{d},
$$

where the meridional pressure gradient is balanced by horizontal viscous dissipation. Pedlosky (2003) called this the hydrostatic layer. The Rossby radius of deformation is given by

$$
L_{d}=\frac{\sqrt{g^{\prime} H}}{2 f},
$$

where $g^{\prime}$ is the reduced gravity. For $A \sim 10 \mathrm{~m}^{2} \mathrm{~s}^{-1}$, $K_{e} \sim 100 \mathrm{~m}^{2} \mathrm{~s}^{-1}$, and $L_{d} \sim 30 \mathrm{~km}$, we get $\delta \sim 10 \mathrm{~km}$, 
(a)

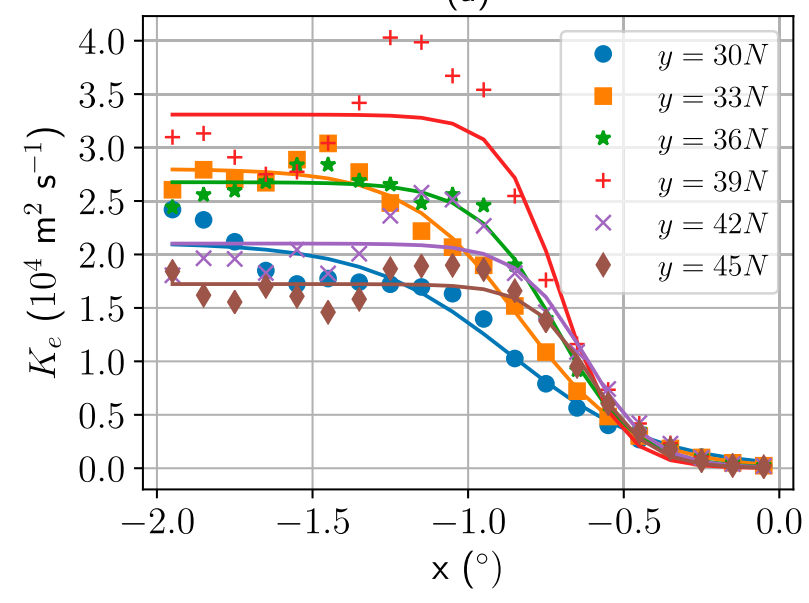

(b)

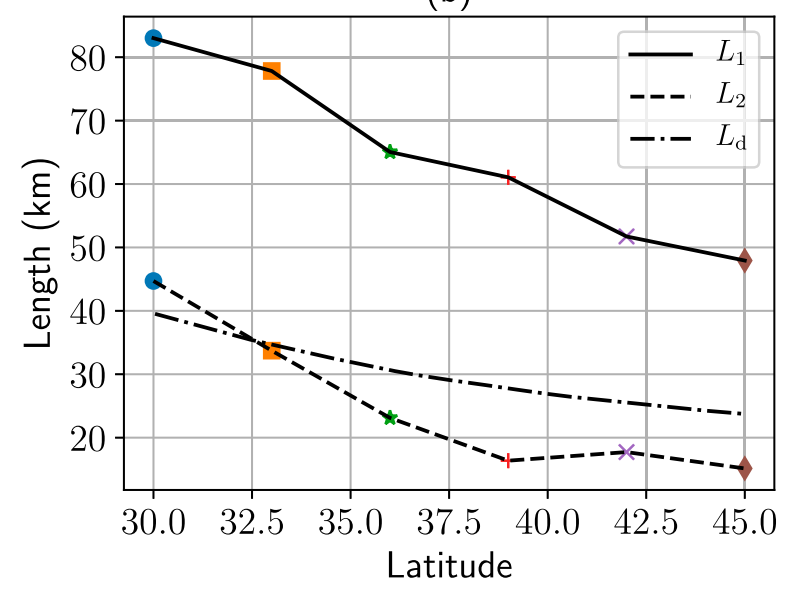

FIG. 14. (a) Zonal variation of $K_{e}$ at various latitudes. (b) Meridional profiles of $L_{1}, L_{2}$, and first baroclinic Rossby deformation radius $L_{d}$.

which is thin compared to the total width of the eastern boundary current. As noted by Cessi and Wolfe (2009) and Cessi et al. (2010), it is possible to avoid explicit consideration of the hydrostatic layer by using an effective boundary condition. To derive this condition, we integrate (34) over the width of the hydrostatic layer:

$$
\hat{u}(0)-\hat{u}(-\delta)+\int_{-\delta}^{0} \hat{v}_{y} d x=\frac{4}{H} \int_{-\delta}^{0} \bar{w}_{i} d x .
$$

Applying the no-normal flow boundary condition and noting that $\delta$ is small leads to the boundary condition

$$
\hat{u}(-\delta)=0 .
$$

Since $\delta$ is small, we make only a small error by applying the boundary condition at $x=0$. The system that governs the flow outside the hydrostatic layer is

$$
\begin{aligned}
-f \hat{v} & =g^{\prime} \bar{\eta}_{x}, \\
f \hat{u} & =g^{\prime} \bar{\eta}_{y}+\frac{4 f K_{e}}{H} \bar{\eta}_{x}-r \hat{v}, \quad \text { and } \\
\nabla \cdot \hat{\mathbf{u}} & =\frac{4}{H} \bar{w}_{i} .
\end{aligned}
$$

Combining (40) with (41) and (42) gives the effective boundary condition on the eastern boundary in terms of $\bar{\eta}$ :

$$
\frac{K_{\mathrm{eff}, E}}{f L_{d}^{2}} \bar{\eta}_{x}+\bar{\eta}_{y}=0
$$

where

$$
K_{\mathrm{eff}, E}=K_{e}(x=0)+r L_{d}^{2} .
$$

A vorticity equation in terms of $\bar{\eta}$ can be derived from the system in (41)-(43):

$$
\left(K_{\mathrm{eff}} \bar{\eta}_{x}\right)_{x}+\beta L_{d}^{2} \bar{\eta}_{x}-\gamma(\bar{\eta}-\theta)=0,
$$

where

$$
K_{\mathrm{eff}}=K_{e}+r L_{d}^{2} .
$$

We seek solutions of the form $\bar{\eta}=\eta^{u}+\eta^{b}, \bar{u}=u^{u}+u^{b}$, and $\bar{v}=v^{u}+v^{b}$, where the superscripts $u$ and $b$ represent unbounded and bounded solutions of the system. The unbounded solution is straightforward and given by assuming zonal derivatives to be zero; that is,

$$
\eta^{u}=\theta, \quad u^{u}=\frac{g^{\prime} \theta_{y}}{f}, \quad v^{u}=0
$$

The bounded solution is given by the solution to

$$
\left(K_{\mathrm{eff}} \eta_{x}^{b}\right)_{x}+\beta L_{d}^{2} \eta_{x}^{b}-\gamma \eta^{b}=0
$$

with the eastern boundary condition

$$
\frac{K_{\mathrm{eff}, E}}{f L_{d}^{2}} \eta_{x}^{b}+\eta_{y}^{b}+\frac{f}{g^{\prime}} u^{u}=0 .
$$

Equation (49) can be numerically solved by using the method of lines. Far to the west, $K_{\text {eff }}=K_{e, I}+r L_{d}^{2}=$ $K_{\text {eff }, W}$ is constant, so (49) becomes

$$
K_{\mathrm{eff}, W} \eta_{x x}^{b}+\beta L_{d}^{2} \eta_{x}^{b}-\gamma \eta^{b}=0
$$

We select a solution that decays to the west by imposing

$$
\eta_{x}^{b}-k_{w} \eta^{b}=0
$$


(a)

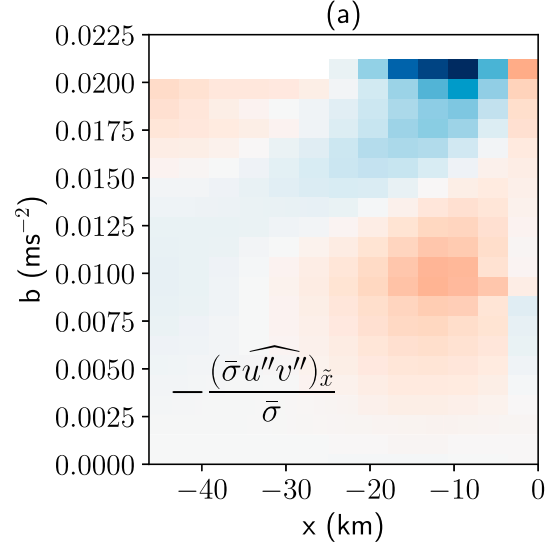

(b)

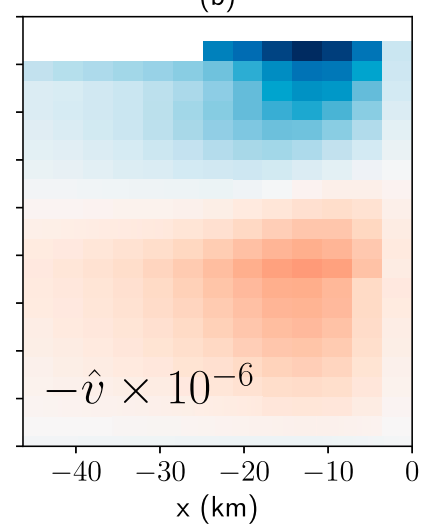

(c)

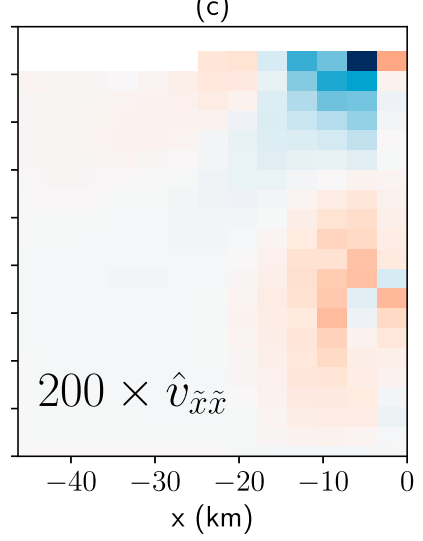

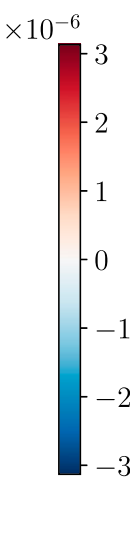

FIG. 15. Zonal sections of (a) Reynolds stress divergence, (b) $-\hat{v} \times 10^{-6}$, and (c) $\hat{v}_{\tilde{x} \tilde{x}} \times 200$ averaged between $35^{\circ}$ and $37^{\circ} \mathrm{N}$ near the eastern boundary.

where

$$
k_{w}=-\frac{\beta L_{d}^{2}}{2 K_{\mathrm{eff}, W}}+\sqrt{\frac{\beta^{2} L_{d}^{4}}{4 K_{\mathrm{eff}, W}^{2}}+\frac{\gamma}{K_{\mathrm{eff}, W}}},
$$

on an artificial western boundary far from the eastern boundary. Equation (49) is solved numerically using SciPy's "solve_bvp" function with the boundary condition in (52) on the western boundary and $\eta^{b}=1$ on the eastern boundary to obtain a zonal profile of $\eta^{b}$. The slope, $k_{e}=\eta_{x}^{b} / \eta^{b}$, is calculated at the eastern boundary from this numerical solution. Substituting $\eta_{x}^{b}=k_{e} \eta^{b}$ in (50), we find

$$
\frac{K_{\mathrm{eff}, E}}{f L_{d}^{2}} k_{e} \eta^{b}+\eta_{y}^{b}+\frac{f}{g^{\prime}} u^{u}=0
$$

which is solved analytically to give the meridional variation of $\eta^{b}$ at the eastern boundary:

$$
\eta^{b}(x=0, y)= \begin{cases}0, & y \leq y_{S}, \\ \frac{s}{\alpha}\left(e^{\alpha\left(y_{S}-y\right)}-1\right), & y_{S} \leq y<y_{N}, \\ {\left[\frac{s}{\alpha}\left(e^{\alpha\left(y_{S}-y_{N}\right)}-1\right)\right] e^{-\alpha\left(y-y_{N}\right)},} & y>y_{N},\end{cases}
$$

where

$$
\alpha=\frac{K_{\mathrm{eff}, E} k_{e}}{f L_{d}^{2}}
$$

represents a meridional inverse length scale over which the bounded solution in the unforced region (north of $y_{N}$ ) decays. We are assuming that $f$ is a constant except when differentiated. This formally restricts the dynamics to slightly smaller than basin scale but does not alter the essential dynamics. The overall bounded solution is obtained by multiplying the zonal and meridional profiles of $\eta^{b}$.

The total solutions $\bar{\eta}, \bar{u}, \bar{v}$, and $\hat{u}$ are shown in Fig. 16 . Negative interface displacement in the southern part of the domain (Fig. 16c), imposed by the forcing, implies warmer (colder) water in the south (north), which flows along the eastern boundary as a geostrophic northward current (Fig. 16c). The northward current is fed by an onshore zonal flow (Fig. 16b), which results from the northward shoaling of the interface. The zonal residual velocity (Fig. 16d) is weaker compared to the mean zonal velocity and even reverses north of the forcing region. This westward flow is a result of the zonal interface gradient [see (A22)] and represents the offshore transport due to eddies.

The zonal sections of currents and interface displacement are shown in Fig. 17. Increasing $L_{1}$, which represents the location of change in diffusivity, broadens the current (Fig. 17d). The sharpness of the change in diffusivity, represented by $L_{2}$, also influences sharpness of the meridional current. Decreasing $L_{2}$ makes the western flank of the current sharper (Fig. 17e).

In the presence of $\beta$, the amplitude of the meridional velocity increases and the peak moves slightly offshore (Figs. 17d-f), while the meridional decay scale of meridional velocity decreases (not shown). The latter is due 

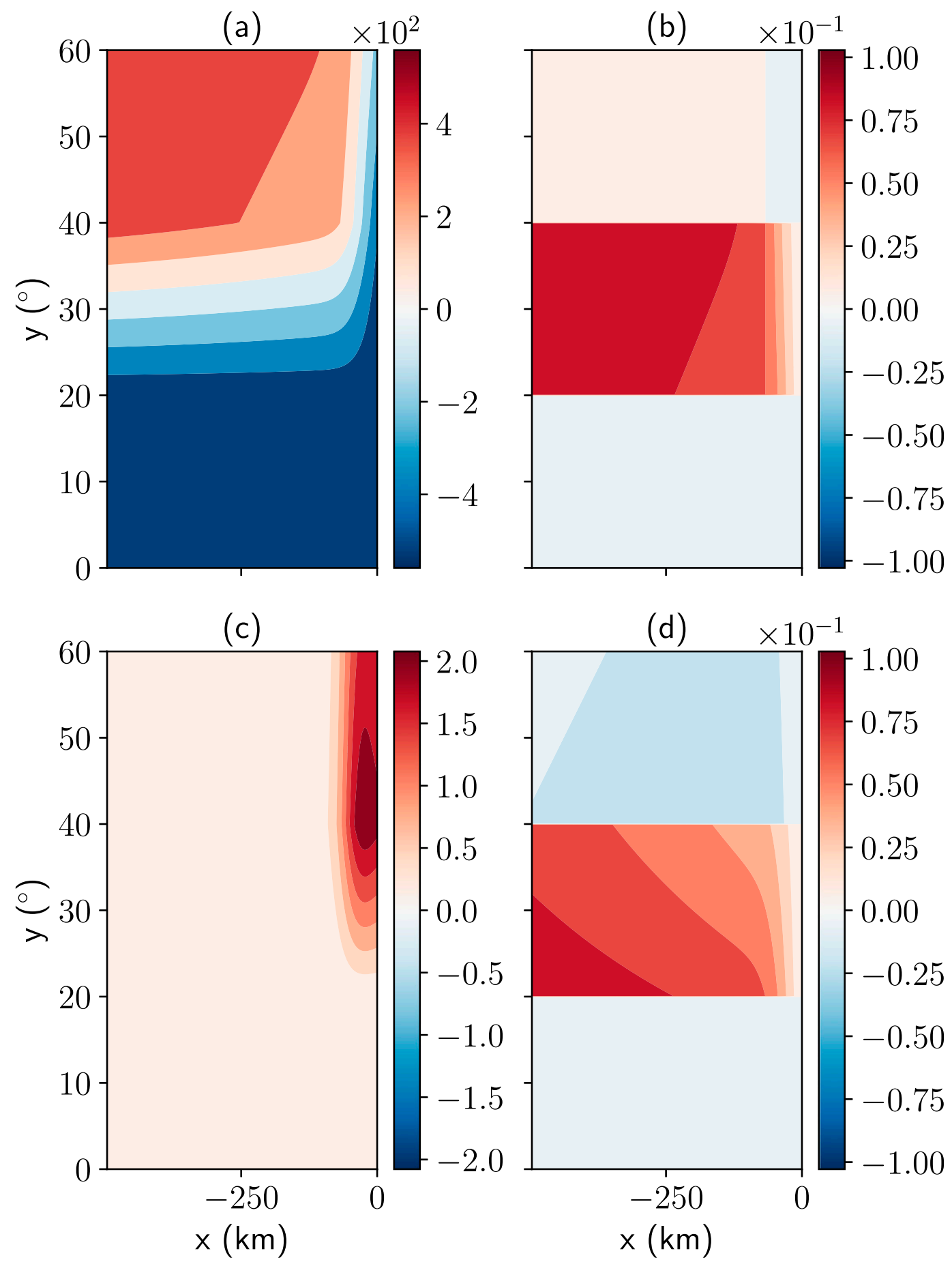

FIG. 16. Plan views of (a) $\bar{\eta}$, (b) $\bar{u}$, (c) $\bar{v}$, and (d) $\hat{u}$ obtained from the two-layer model. Interface displacement (m) and velocities $\left(\mathrm{m} \mathrm{s}^{-1}\right)$ are represented. The values of tunable parameters used are $K_{e, I}=25000 \mathrm{~m}^{2} \mathrm{~s}^{-1}, r=10^{-6} \mathrm{~s}^{-1}$, $s=5 \times 10^{-4}, f=8.57 \times 10^{-5} \mathrm{~s}^{-1}, \quad \beta=1.8 \times 10^{-11}(\mathrm{~m} \mathrm{~s})^{-1}, H=1500 \mathrm{~m}, \quad L_{d}=30 \mathrm{~km}, \quad L_{1}=100 \mathrm{~km}, \quad L_{2}=30 \mathrm{~km}$, $\gamma^{-1}=120$ days, $\eta_{\mathrm{s}}=-560 \mathrm{~m}, y_{S}=20^{\circ} \mathrm{N}$, and $y_{N}=40^{\circ} \mathrm{N}$. The domain extends zonally from -4000 to $0 \mathrm{~km}$ and meridionally from $0^{\circ}$ to $60^{\circ} \mathrm{N}$. 
(a)

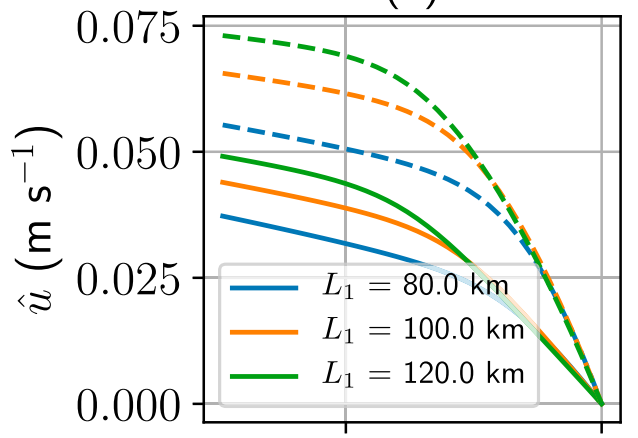

(d)

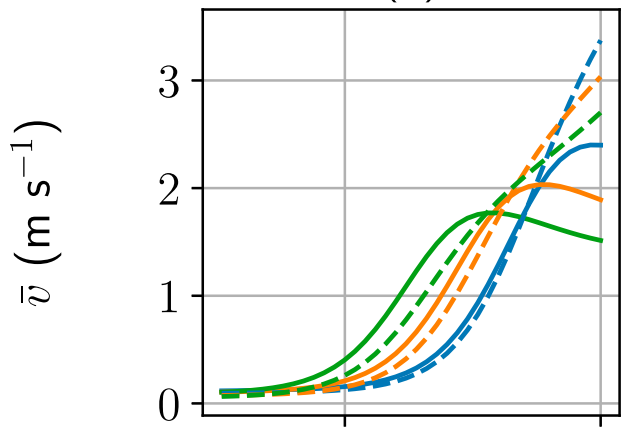

(g)

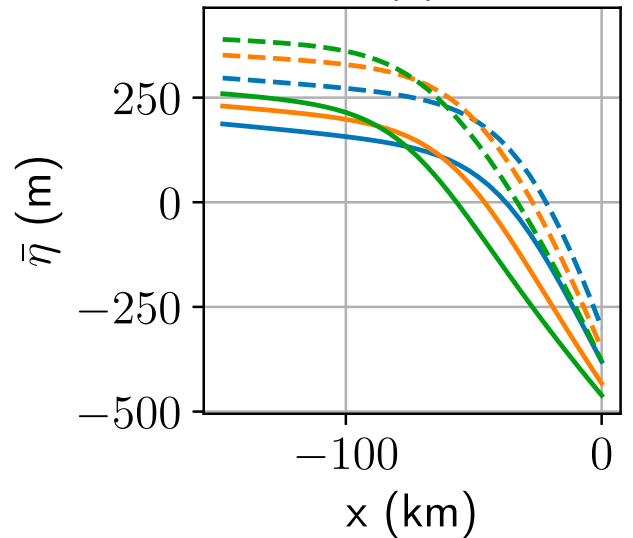

(b)

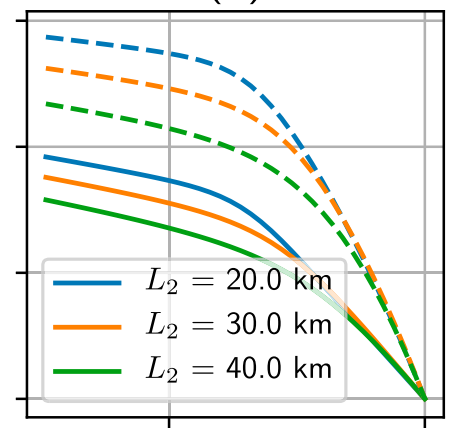

(e)

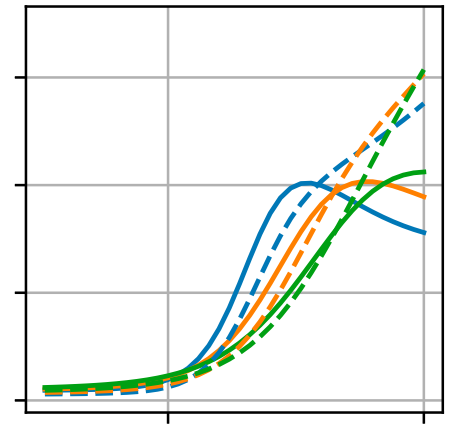

(h)

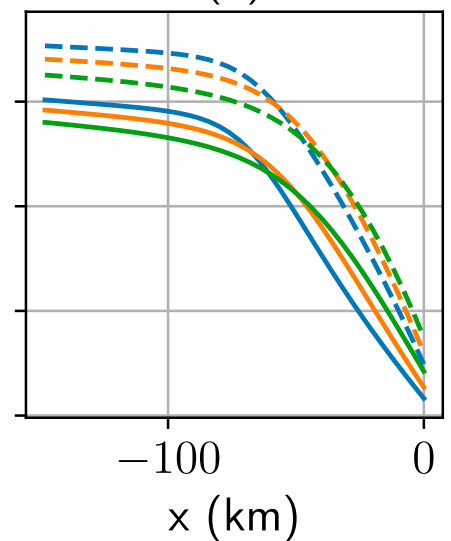

(c)

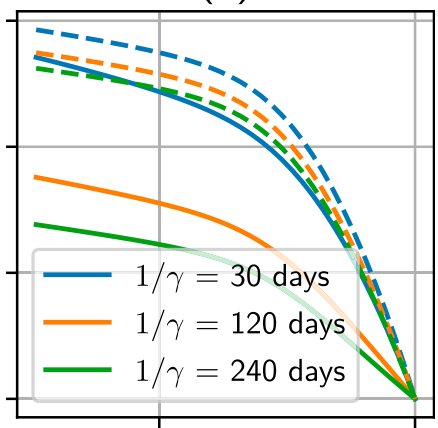

(f)

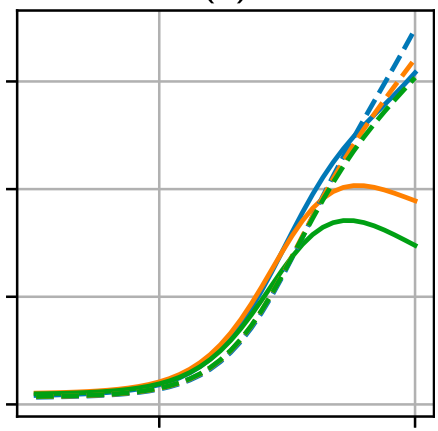

(i)

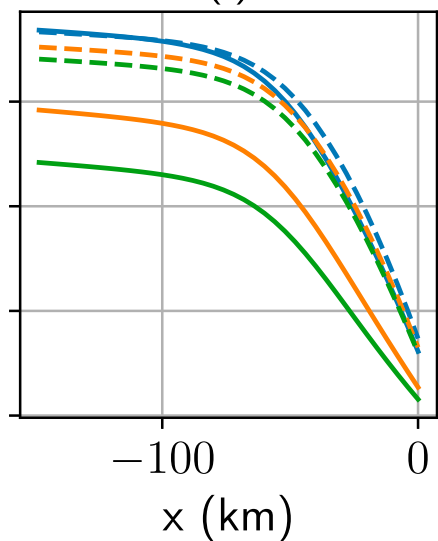

FIG. 17. Zonal profiles of (a)-(c) $\hat{u}$, (d)-(f) $\bar{v}$, and (g)-(i) $\bar{\eta}$ at $40^{\circ} \mathrm{N}$ are shown. Variation with (left) $L_{1}$, (center) $L_{2}$, and (right) $\gamma$ is shown. Solid and dashed lines indicate profiles with and without the $\beta$ effect, respectively. Values of tunable parameters are as in Fig. 16.

to the dependence of the meridional decay scale $\alpha$ on $k_{e}$ [see (56)], which decreases when $\beta$ is present. The former is due to Rossby waves allowing more of the PV anomaly to leak offshore. Large values of $\gamma$ tend to overwhelm the effect of $\beta$. Full analysis of the sensitivity of this simple model is beyond the scope of this study. However, the fact that such a simple model is able to recreate the circulation observed in an eddy-resolving model lends credence to the theory that zonal variation of eddy diffusivity captures a narrow eastern boundary current.
The physics of the EBC is straightforward. The form drag term acts like a horizontal diffusion of PV (e.g., Treguier et al. 1997; Greatbatch 1998; McDougall and McIntosh 2001). If the diffusivity varies in space, the PV gradient must vary inversely to the diffusivity to ensure continuity of the PV flux (if all other things are equal). Since the diffusivity is smaller near the boundary than offshore, the PV gradient must be larger near the coast. The PV gradient is proportional to the curvature and vertical shear of the meridional velocity, so the meridional velocity must itself be larger near the eastern boundary. 
An alternate interpretation can be made in terms of vertical momentum mixing, since the form drag term represents a vertical transfer of momentum. Vertical momentum mixing is small near the boundary, which allows the northward and southward EBCs to remain distinct. Further offshore, vertical mixing of momentum is strong, resulting in a vertical homogenization of meridional momentum. Since the northward and southward EBCs have nearly equal and opposite momentum, the homogeneous state has very small meridional velocity.

\section{Summary}

We simulate an eastern boundary current in an idealized eddy-resolving model forced by large-scale buoyancy forcing. This current has a baroclinic structure, with poleward flow stacked above equatorward flow, and sheds predominantly anticyclonic eddies at the surface. While sloping bottom topography and coastal shape has been argued to play an important role in the formation of anticyclonic eddies in poleward eastern boundary currents (Pelland et al. 2013; Molemaker et al. 2015; Southwick et al. 2016), this study shows that anticyclones are also formed in the absence of these effects. The alongshore currents are sufficiently strong that eddy formation is spontaneous and the prevalence of anticyclones at the surface is due to their warm cores, resulting from entrainment of buoyant water transported northward in the boundary current.

These eddies have a profound effect on the residual circulation and budgets of momentum and buoyancy. The westward zonal eddy-induced velocity overcomes the eastward mean velocity, resulting in a residual circulation that has the opposite sense as the mean in the upper water column. This broadens the downwelling limb of the residual-mean zonal overturning circulation so that diapycnal downwelling is evenly distributed across the interior instead of being concentrated near the eastern boundary. In the momentum budget, the efficiency of form drag increases dramatically $50-100 \mathrm{~km}$ from the eastern boundary and homogenizes momentum vertically. The maximum form drag is displaced seaward of the maximum current velocity, so the momentum flux cycle in the EBC involves offshore transport near the surface by Reynolds stresses, downward transport by form drag, and finally onshore transport by Reynolds stresses at the level of the undercurrent. The overall effect of the EP flux divergence is to mix momentum vertically but with an offshore excursion.

The form drag acts like a vertical viscosity (or horizontal diffusion of PV) with a coefficient that increases offshore before saturating at a large value. The effect of form drag is small near the boundary, so momentum is not homogenized vertically and there are two distinct jets. Away from the boundary, the form drag strengthens and homogenizes the two jets, effectively erasing the boundary currents. Thus, the scale over which form drag increases offshore sets the scale of the EBC. The diffusivity associated with the form drag and its zonal variation serve as good diagnostic tools to understand the cross-shore structure of the eastern boundary currents. A more complete theory would predict the zonal variation of the diffusivity and is the subject of ongoing research.

Acknowledgments. This research was funded by the NSF (OCE-1559065). This work used the Extreme Science and Engineering Discovery Environment (XSEDE) Stampede cluster at the Texas Advanced Computing Center through the allocation TG-OCE160008. The authors are thankful to the helpful and responsive team at NOAA/GFDL involved in developing the Modular Ocean Model 6 (MOM6) as well as to the Python community for providing free and open-source tools like Jupyter, Numpy, SciPy, Matplotlib, and Xarray.

The authors are grateful to the two anonymous reviewers whose feedback directly led to improvements in this manuscript. Finally, the authors thank Michael Spall for several helpful suggestions and comments.

\section{APPENDIX}

\section{Two-Layer Model for the Residual Flow}

We start out with the unaveraged two-layer system (the tildes on coordinates have been dropped):

$$
\begin{aligned}
\frac{D u_{n}}{D t}-f v_{n} & =-m_{n, x}, \\
\frac{D v_{n}}{D t}+f u_{n} & =-m_{n, y}, \quad \text { and } \\
h_{n, t}+\nabla \cdot h_{n} \mathbf{u}_{n} & =(-1)^{n+1} w_{i} .
\end{aligned}
$$

We employ the rigid lid approximation, so that $h_{1}+h_{2}=H$ and $m_{2}=m_{1}+g^{\prime} \eta$, where $H$ is the total depth, and $\eta$ is the height of the interface between the two layers. Note that we are ignoring any momentum flux due to the (presumed small) interfacial velocity $w_{i}$, so

$$
\frac{D}{D t}=\frac{\partial}{\partial t}+u \frac{\partial}{\partial x}+v \frac{\partial}{\partial y}
$$

Multiplying the momentum equation by thickness and averaging gives 
$\frac{D^{\sharp} \hat{u}_{n}}{D t}-f \hat{v}_{n}=-\bar{m}_{n, x}-\frac{\overline{h_{n}^{\prime} m_{n, x}^{\prime}}}{\bar{h}_{n}}-\bar{h}_{n}^{-1} \nabla \cdot \mathbf{J}^{u}$,

$\frac{D^{\ddagger} \hat{v}_{n}}{D t}+f \hat{u}_{n}=-\bar{m}_{n, y}-\frac{\overline{h_{n}^{\prime} m_{n, y}^{\prime}}}{\bar{h}_{n}}-\bar{h}_{n}^{-1} \nabla \cdot \mathbf{J}^{v}, \quad$ and

$\bar{h}_{n, t}+\nabla \cdot \bar{h}_{n} \hat{\mathbf{u}}_{n}=(-1)^{n+1} \bar{w}_{i}$,

where

$$
\frac{D^{\sharp}}{D t}=\frac{\partial}{\partial t}+\hat{u} \frac{\partial}{\partial x}+\hat{v} \frac{\partial}{\partial y},
$$

and

$$
\mathbf{J}^{\theta}=\bar{h}_{n} \widehat{u^{\prime \prime} \theta^{\prime \prime}} \mathbf{i}+\bar{h}_{n} \widehat{v^{\prime \prime} \theta^{\prime \prime}} \mathbf{j}
$$

The Reynolds stresses are parameterized as a combination of Laplacian friction and linear drag with uniform viscosity $A$ and drag coefficient $r$, respectively. The form drag terms are parameterized assuming eddies are geostrophic and then invoking a flux-gradient relationship:

$$
\begin{aligned}
& \overline{h_{n}^{\prime} m_{n, x}^{\prime}} \approx f \overline{h_{n}^{\prime} v_{n}^{\prime}} \sim-f K_{e} \bar{h}_{n, y}, \quad \text { and } \\
& \overline{h_{n}^{\prime} m_{n, y}^{\prime}} \approx-f \overline{h_{n}^{\prime} u_{n}^{\prime}} \sim f K_{e} \bar{h}_{n, x} .
\end{aligned}
$$

With this parameterization, the residual velocities are

$$
\hat{\mathbf{u}}_{n}=\overline{\mathbf{u}}_{n}+\frac{\overline{h^{\prime} \mathbf{u}_{n}^{\prime}}}{\bar{h}} \sim \overline{\mathbf{u}}_{n}-K_{e} \frac{\nabla \bar{h}_{n}}{\bar{h}_{n}} .
$$

The parameterized system is

$$
\begin{aligned}
& \frac{D^{\ddagger} \hat{u}_{n}}{D t}-f \hat{v}_{n}=-\bar{m}_{n, x}+f K_{e} \frac{\bar{h}_{n, y}}{\bar{h}_{n}}+\frac{A}{\bar{h}_{n}} \nabla \cdot h_{n} \nabla \hat{u}_{n}-r \hat{u}_{n}, \\
& \frac{D^{\ddagger} \hat{v}_{n}}{D t}+f \hat{u}_{n}=-\bar{m}_{n, y}-f K_{e} \frac{\bar{h}_{n, x}}{\bar{h}_{n}}+\frac{A}{\bar{h}_{n}} \nabla \cdot h_{n} \nabla \hat{v}_{n}-r \hat{v}_{n},
\end{aligned}
$$

$\bar{h}_{t}+\nabla \cdot \bar{h}_{n} \hat{\mathbf{u}}_{n}=(-1)^{n+1} \bar{w}_{i}$.

We now assume a steady state and linearize about resting depths $H_{1}$ and $H_{2}$, assumed to be equal to $H / 2$. The linearized system is

$$
\begin{aligned}
-f \hat{v}_{n} & =-\bar{m}_{n, x}+2 f K_{e} \frac{\bar{h}_{n, y}}{H}+A \nabla^{2} \hat{u}_{n}-r \hat{u}_{n}, \\
f \hat{u}_{n} & =-\bar{m}_{n, y}-2 f K_{e} \frac{\bar{h}_{n, x}}{H}+A \nabla^{2} \hat{v}_{n}-r \hat{v}_{n}, \quad \text { and }
\end{aligned}
$$

$$
\nabla \cdot \hat{\mathbf{u}}_{n}=(-1)^{n+1} \frac{2}{H} \bar{w}_{i} .
$$

Subtracting the layer 2 equations from layer 1 equations gives

$$
\begin{aligned}
-f \hat{v} & =g^{\prime} \bar{\eta}_{x}-\frac{4 f K_{e}}{H} \bar{\eta}_{y}+A \nabla^{2} \hat{u}-r \hat{u}, \\
f \hat{u} & =g^{\prime} \bar{\eta}_{y}+\frac{4 f K_{e}}{H} \bar{\eta}_{x}+A \nabla^{2} \hat{v}-r \hat{v}, \quad \text { and } \\
\nabla \cdot \hat{\mathbf{u}} & =\frac{4}{H} \bar{w}_{i}
\end{aligned}
$$

where $\mathbf{u}=\mathbf{u}_{1}-\mathbf{u}_{2}$. We can write this system in terms of Eulerian-mean quantities by noting that

$$
\hat{\mathbf{u}}=\overline{\mathbf{u}}+\frac{4 K_{e}}{H} \nabla \bar{\eta} .
$$

The Eulerian-mean system is therefore

$$
\begin{aligned}
-f \bar{v} & =g^{\prime} \bar{\eta}_{x}+A \nabla^{2} \bar{u}-r \bar{u}, \\
f \bar{u} & =g^{\prime} \bar{\eta}_{y}+A \nabla^{2} \bar{v}-r \bar{v}, \quad \text { and } \\
\nabla \cdot \overline{\mathbf{u}} & =\frac{4}{H}\left(\bar{w}_{i}-\nabla \cdot K_{e} \nabla \bar{\eta}\right) .
\end{aligned}
$$

Here, we have neglected products of $K_{e}$ with $A$ and $r$ in the friction terms. Ignoring the linear drag terms, this system is very similar to that used by Pedlosky and Spall (2005), except for a factor of 2 difference in the definition of $H$ and a different interpretation of the Laplacian term appearing in the continuity equation.

\section{REFERENCES}

Adcroft, A., and R. Hallberg, 2006: On methods for solving the oceanic equations of motion in generalized vertical coordinates. Ocean Modell., 11, 224-233, https://doi.org/10.1016/ j.ocemod.2004.12.007.

,$- \ldots$, and M. Harrison, 2008: A finite volume discretization of the pressure gradient force using analytic integration. Ocean Modell., 22, 106-113, https://doi.org/10.1016/j.ocemod.2008.02.001.

Bates, M., R. Tulloch, J. Marshall, and R. Ferrari, 2014: Rationalizing the spatial distribution of mesoscale eddy diffusivity in terms of mixing length theory. J. Phys. Oceanogr., 44, 15231540, https://doi.org/10.1175/JPO-D-13-0130.1.

Benthuysen, J., R. Furue, J. P. McCreary, N. L. Bindoff, and H. E. Phillips, 2014: Dynamics of the Leeuwin Current: Part 2. Impacts of mixing, friction, and advection on a buoyancy-driven eastern boundary current over a shelf. Dyn. Atmos. Oceans, 65, 39-63, https://doi.org/10.1016/j.dynatmoce.2013.10.004.

Cessi, P., and C. L. Wolfe, 2009: Eddy-driven buoyancy gradients on eastern boundaries and their role in the thermocline. J. Phys. Oceanogr., 39, 1595-1614, https://doi.org/10.1175/ 2009JPO4063.1. 
$\longrightarrow$, and - 2013: Adiabatic eastern boundary currents. J. Phys. Oceanogr., 43, 1127-1149, https://doi.org/10.1175/JPO-D-120211.1.

$\longrightarrow, \ldots$, and B. C. Ludka, 2010: Eastern-boundary contribution to the residual and meridional overturning circulations. J. Phys. Oceanogr., 40, 2075-2090, https://doi.org/10.1175/ 2010JPO4426.1.

Chelton, D. B., M. G. Schlax, and R. M. Samelson, 2011: Global observations of nonlinear mesoscale eddies. Prog. Oceanogr., 91, 167-216, https://doi.org/10.1016/j.pocean.2011.01.002.

Connolly, T. P., B. M. Hickey, I. Shulman, and R. E. Thomson, 2014: Coastal trapped waves, alongshore pressure gradients, and the California Undercurrent. J. Phys. Oceanogr., 44, 319342, https://doi.org/10.1175/JPO-D-13-095.1.

Cresswell, G. R., and T. J. Golding, 1980: Observations of a southflowing current in the southeastern Indian Ocean. Deep-Sea Res., 27A, 449-466, https://doi.org/10.1016/0198-0149(80) 90055-2.

Csanady, G. T., 1985: "Pycnobathic" currents over the upper continental slope. J. Phys. Oceanogr., 15, 306-315, https://doi.org/ 10.1175/1520-0485(1985)015<0306:COTUCS $>2.0 . C O ; 2$.

De Szoeke, R. A., and A. F. Bennett, 1993: Microstructure fluxes across density surfaces. J. Phys. Oceanogr., 23, 2254-2264, https://doi.org/10.1175/1520-0485(1993)023<2254:MFADS> 2.0.CO;2.

de Verdière, C. A., 1988: Buoyancy driven planetary flows. J. Mar. Res., 46, 215-265, https://doi.org/10.1357/002224088785113667.

Fang, F., and R. Morrow, 2003: Evolution, movement and decay of warm-core Leeuwin Current eddies. Deep-Sea Res. II, 50, 2245-2261, https://doi.org/10.1016/S0967-0645(03)00055-9.

Ferrari, R., and M. Nikurashin, 2010: Suppression of eddy diffusivity across jets in the Southern Ocean. J. Phys. Oceanogr., 40, 1501-1519, https://doi.org/10.1175/2010JPO4278.1.

Ferreira, D., and J. Marshall, 2006: Formulation and implementation of a "residual-mean" ocean circulation model. Ocean Modell., 13, 86-107, https://doi.org/10.1016/j.ocemod.2005.12.001.

Frouin, R., A. F. G. Fiúza, I. Ambar, and T. J. Boyd, 1990: Observations of a poleward surface current off the coasts of Portugal and Spain during winter. J. Geophys. Res., 95, 679691, https://doi.org/10.1029/JC095iC01p00679.

Furue, R., J. P. McCreary, J. Benthuysen, H. E. Phillips, and N. L. Bindoff, 2013: Dynamics of the Leeuwin Current: Part 1. Coastal flows in an inviscid, variable-density, layer model. Dyn. Atmos. Oceans, 63, 24-59, https://doi.org/ 10.1016/j.dynatmoce.2013.03.003.

Gent, P. R., J. Willebrand, T. J. McDougall, and J. C. McWilliams, 1995: Parameterizing eddy-induced tracer transports in ocean circulation models. J. Phys. Oceanogr., 25, 463-474, https:// doi.org/10.1175/1520-0485(1995)025<0463:PEITTI>2.0.CO;2.

Godfrey, J. S., and A. J. Weaver, 1991: Is the Leeuwin Current driven by Pacific heating and winds? Prog. Oceanogr., 27, 225 272, https://doi.org/10.1016/0079-6611(91)90026-I.

Greatbatch, R. J., 1998: Exploring the relationship between eddyinduced transport velocity, vertical momentum transfer, and the isopycnal flux of potential vorticity. J. Phys. Oceanogr., 28, 422-432, https://doi.org/10.1175/1520-0485(1998)028<0422: ETRBEI $>2.0 . \mathrm{CO} ; 2$.

__ , and K. G. Lamb, 1990: On parameterizing vertical mixing of momentum in non-eddy resolving ocean models. J. Phys. Oceanogr., 20, 1634-1637, https://doi.org/10.1175/1520-0485 (1990) $020<1634$ :OPVMOM $>2.0$. CO; 2 .

Griffies, S. M., and R. W. Hallberg, 2000: Biharmonic friction with a Smagorinsky-like viscosity for use in large-scale eddy-permitting ocean models. Mon. Wea. Rev., 128, 2935-2946, https://doi.org/10.1175/1520-0493(2000)128<2935: BFWASL $>2.0 . \mathrm{CO} ; 2$.

Griffiths, R. W., and A. F. Pearce, 1985: Instability and eddy pairs on the Leeuwin Current south of Australia. Deep-Sea Res., 32A, 1511-1534, https://doi.org/10.1016/0198-0149(85)90101-3.

Hallberg, R., 2000: Time integration of diapycnal diffusion and Richardson number-dependent mixing in isopycnal coordinate ocean models. Mon. Wea. Rev., 128, 1402-1419, https://doi.org/ 10.1175/1520-0493(2000)128<1402:TIODDA > 2.0.CO;2.

— , and A. Adcroft, 2009: Reconciling estimates of the free surface height in Lagrangian vertical coordinate ocean models with mode-split time stepping. Ocean Modell., 29, 15-26, https://doi.org/10.1016/j.ocemod.2009.02.008.

Haney, R. L., 1971: Surface thermal boundary condition for ocean circulation models. J. Phys. Oceanogr., 1, 241-248, https:// doi.org/10.1175/1520-0485(1971)001<0241:STBCFO > 2.0.CO;2.

Haynes, R., and E. D. Barton, 1990: A poleward flow along the Atlantic coast of the Iberian Peninsula. J. Geophys. Res., 95, 11 425-11 441, https://doi.org/10.1029/JC095iC07p11425.

Hickey, B. M., 1979: The California Current System-Hypotheses and facts. Prog. Oceanogr., 8, 191-279, https://doi.org/10.1016/ 0079-6611(79)90002-8.

Klocker, A., and R. Abernathey, 2014: Global patterns of mesoscale eddy properties and diffusivities. J. Phys. Oceanogr., 44, 1030-1046, https://doi.org/10.1175/JPO-D-13-0159.1.

_, R. Ferrari, and J. H. LaCasce, 2012: Estimating suppression of eddy mixing by mean flows. J. Phys. Oceanogr., 42, 15661576, https://doi.org/10.1175/JPO-D-11-0205.1.

Kurian, J., F. Colas, X. Capet, J. C. McWilliams, and D. B. Chelton, 2011: Eddy properties in the California Current System. J. Geophys. Res., 116, C08027, https://doi.org/10.1029/ 2010JC006895.

Maddison, J. R., and D. P. Marshall, 2013: The Eliassen-Palm flux tensor. J. Fluid Mech., 729, 69-102, https://doi.org/10.1017/ jfm.2013.259.

Marotzke, J., 1997: Boundary mixing and the dynamics of threedimensional thermohaline circulations. J. Phys. Oceanogr., 27, 1713-1728, https://doi.org/10.1175/1520-0485(1997)027<1713: BMATDO $>2.0 . \mathrm{CO} ; 2$.

McCreary, J. P., 1981: A linear stratified ocean model of the coastal undercurrent. Philos. Trans. Roy. Soc. London, 302A, 385413, https://doi.org/10.1098/rsta.1981.0176.

_ S. R. Shetye, and P. K. Kundu, 1986: Thermohaline forcing of eastern boundary currents: With application to the circulation off the west coast of Australia. J. Mar. Res., 44, 71-92, https:// doi.org/10.1357/002224086788460184.

, Y. Fukamachi, and P. Lu, 1992: A nonlinear mechanism for maintaining coastally trapped eastern boundary currents. J. Geophys. Res., 97, 5677-5692, https://doi.org/10.1029/ 92JC00035.

McDougall, T. J., and P. C. McIntosh, 2001: The temporal-residualmean velocity. Part II: Isopycnal interpretation and the tracer and momentum equations. J. Phys. Oceanogr., 31, 1222-1246, https://doi.org/10.1175/1520-0485(2001)031<1222:TTRMVP> 2.0.CO;2

Molemaker, M. J., J. C. McWilliams, and W. K. Dewar, 2015: Submesoscale instability and generation of mesoscale anticyclones near a separation of the California Undercurrent. J. Phys. Oceanogr., 45, 613-629, https://doi.org/10.1175/JPO-D-13-0225.1.

Nikurashin, M., and G. Vallis, 2011: A theory of deep stratification and overturning circulation in the ocean. J. Phys. Oceanogr., 41, 485-502, https://doi.org/10.1175/2010JPO4529.1. 
Okubo, A., 1970: Horizontal dispersion of floatable particles in the vicinity of velocity singularities such as convergences. DeepSea Res. Oceanogr. Abstr., 17, 445-454, https://doi.org/10.1016/ 0011-7471(70)90059-8.

Park, Y.-G., and K. Bryan, 2001: Comparison of thermally driven circulations from a depth-coordinate model and an isopycnallayer model. Part II: The difference and structure of the circulations. J. Phys. Oceanogr., 31, 2612-2624, https://doi.org/ 10.1175/1520-0485(2001)031<2612: COTDCF >2.0.CO;2.

Pedlosky, J., 2003: Thermally driven circulations in small oceanic basins. J. Phys. Oceanogr., 33, 2333-2340, https://doi.org/ 10.1175/1520-0485(2003)033<2333:TDCISO > 2.0.CO;2.

—, and M. A. Spall, 2005: Boundary intensification of vertical velocity in a $\beta$-plane basin. J. Phys. Oceanogr., 35, 2487-2500, https://doi.org/10.1175/JPO2832.1.

Peliz, A., J. Dubert, D. B. Haidvogel, and B. Le Cann, 2003: Generation and unstable evolution of a density-driven eastern poleward current: The Iberian Poleward Current. J. Geophys. Res., 108, 3268, https://doi.org/10.1029/2002JC001443.

Pelland, N. A., C. C. Eriksen, and C. M. Lee, 2013: Subthermocline eddies over the Washington continental slope as observed by seagliders, 2003-09. J. Phys. Oceanogr., 43, 2025-2053, https:// doi.org/10.1175/JPO-D-12-086.1.

Pingree, R. D., and B. Le Cann, 1992: Three anticyclonic slope water oceanic eddies (SWODDIES) in the Southern Bay of Biscay in 1990. Deep-Sea Res., 39A, 1147-1175, https://doi.org/ 10.1016/0198-0149(92)90062-X.

Saenz, J. A., Q. Chen, and T. Ringler, 2015: Prognostic residual mean flow in an ocean general circulation model and its relation to prognostic Eulerian mean flow. J. Phys. Oceanogr., 45, 2247-2260, https://doi.org/10.1175/JPO-D-15-0024.1.

Schloesser, F., 2014: A dynamical model for the Leeuwin Undercurrent. J. Phys. Oceanogr., 44, 1798-1810, https://doi.org/ 10.1175/JPO-D-13-0226.1.

, R. Furue, J. P. McCreary, and A. Timmermann, 2012: Dynamics of the Atlantic meridional overturning circulation. Part 1: Buoyancy-forced response. Prog. Oceanogr., 101, 33-62, https://doi.org/10.1016/j.pocean.2012.01.002.
Southwick, O. R., E. R. Johnson, and N. R. McDonald, 2016: A simple model for sheddies: Ocean eddies formed from shed vorticity. J. Phys. Oceanogr., 46, 2961-2979, https://doi.org/ 10.1175/JPO-D-15-0251.1.

Spall, M. A., and R. S. Pickart, 2001: Where does dense water sink? A subpolar gyre example. J. Phys. Oceanogr., 31, 810-826, https://doi.org/10.1175/1520-0485(2001)031<0810:WDDWSA > 2.0.CO;2.

Sumata, H., and A. Kubokawa, 2001: Numerical study of eastern boundary ventilation and its effects on the thermocline structure. J. Phys. Oceanogr., 31, 3002-3019, https:// doi.org/10.1175/1520-0485(2001)031<3002:NSOEBV> 2.0.CO;2.

Thompson, R. O. R. Y., 1984: Observations of the Leeuwin Current off Western Australia. J. Phys. Oceanogr., 14, 623-628, https://doi.org/10.1175/1520-0485(1984)014<0623:OOTLCO > 2.0.CO;2.

Treguier, A. M., I. M. Held, and V. D. Larichev, 1997: Parameterization of quasigeostrophic eddies in primitive equation ocean models. J. Phys. Oceanogr., 27, 567-580, https://doi.org/10.1175/ 1520-0485(1997)027<0567:POQEIP > 2.0.CO;2.

Weaver, A. J., and J. H. Middleton, 1989: On the dynamics of the Leeuwin Current. J. Phys. Oceanogr., 19, 626-648, https://doi.org/10.1175/1520-0485(1989)019<0626:OTDOTL > 2.0.CO;2.

— and - 1990: An analytic model for the Leeuwin Current off Western Australia. Cont. Shelf Res., 10, 105-122, https:// doi.org/10.1016/0278-4343(90)90025-H.

Weiss, J., 1991: The dynamics of enstrophy transfer in twodimensional hydrodynamics. Physica D, 48, 273-294, https:// doi.org/10.1016/0167-2789(91)90088-Q.

Wolfe, C. L., and P. Cessi, 2010: What sets the strength of the middepth stratification and overturning circulation in eddying ocean models? J. Phys. Oceanogr., 40, 1520-1538, https:// doi.org/10.1175/2010JPO4393.1.

Young, W. R., 2012: An exact thickness-weighted average formulation of the Boussinesq equations. J. Phys. Oceanogr., 42, 692-707, https://doi.org/10.1175/JPO-D-11-0102.1. 